\title{
ON FORWARD AND INVERSE UNCERTAINTY QUANTIFICATION FOR MODELS INVOLVING HYSTERESIS OPERATORS
}

\author{
Olaf Klein $^{1, *}$, Daniele Davino ${ }^{2}$ and Ciro Visone ${ }^{3}$
}

\begin{abstract}
Parameters within hysteresis operators modeling real world objects have to be identified from measurements and are therefore subject to corresponding errors. To investigate the influence of these errors, the methods of Uncertainty Quantification (UQ) are applied. Results of forward UQ for a play operator with a stochastic yield limit are presented. Moreover, inverse UQ is performed to identify the parameters in the weight function in a Prandtl-Ishlinskil operator and the uncertainties of these parameters.
\end{abstract}

Mathematics Subject Classification. 47J40,60H30.

Received December 17, 2018. Accepted March 29, 2020.

\section{UNCERTAINTIES IN MODELS WITH HYSTERESIS OPERATORS AND UNCERTAINTY QUANTIFICATION}

Considering, e.g., magnetization, piezo-electric effects, elasto-plastic behavior, or magnetostrictive materials, one has to take into account hysteresis effects. Many models involve therefore hysteresis operators. The parameters in the models are identified using results from measurements, sometimes performed only for some sample specimens but also used for other specimens.

The parameters in the hysteresis operators are therefore also subject to uncertainties. We apply the methods of Uncertainty Quantification (UQ), see, e.g., [14, 15], to deal with these uncertainties, i.e., we describe them by introducing appropriate random variables modeling the corresponding information/assumptions/beliefs and use probability theory to describe and determine the influence of the uncertainties.

In this paper, we present results of elementary Forward Uncertainty Quantification. During forward UQ, one starts from the random variable representing the value(s) of the considered parameter(s) in the model and considers the resulting model output as random variable. This allows to compute properties like expected value, variation, probabilities for outputs entering some interval, credible intervals, and other Quantities of Interest (QoI). Moreover, we will also present a brief example of Inverse UQ, i.e., of using (further) data and measurements, to determine (reduce/adapt) the uncertainty of the parameter(s), i.e., to determine a (new) random variable taking into account the (new) information, and use the (new) random variable to represent the parameter(s) afterwards.

Keywords and phrases: Hysteresis, uncertainty quantification.

1 Weierstrass Institute (WIAS), Mohrenstr. 39, 10117 Berlin, Germany.

2 Università degli Studi del Sannio, P.zza Roma, 21 - 82100 Benevento, Italy.

3 Università di Napoli Federico II, Via Claudio, 21- 80125 Napoli, Italy.

* Corresponding author: olaf.klein@wias-berlin.de 
Forward UQ is presented, for example, in [19] for the Bouc-Wen hysteresis model and in [10] for the Davidenkov model with simplified loading-reloading rules.

Forward and inverse UQ can be found for example in [11] for a model involving the stop operator and in [16] for a model involving a hysteretic multi scale formulation.

Inverse UQ for a Prandtl-Ishlinskiĭ operator of stop type can be found in [13].

The plan of the paper is the following: In Section 2, the play operator with deterministic data is recalled and a method to efficiently compute the values of the play operators for all possible yield limits is introduced. In Section 3, we will consider the play operator if the yield limit is not know exactly such that we represented it by a random variable and perform forward UQ to determine the evolution of the uncertainty. In Section 4, we will recall the generalized Prandtl-Ishlinskil operator with a parameterized weight function as in [4], introduce the corresponding initial loading curve and present equations that are satisfied by this curve. In Section 5, we will consider results of measurements and will try to identify the values of the parameters and extract information about their uncertainties, i.e., we perform inverse UQ. In the first attempt, we extract several approximations of the initial curve from the measurements, use the equations introduced in Section 4 to compute for each approximation a corresponding pair of parameters and investigate the resulting set of pairs. In the second attempt, Bayes' Theorem is applied.

\section{THE PLAY OPERATOR WITH DETERMINISTIC DATA}

An important example for an hysteresis operator is the play operator, see, e.g., [3, 8, 9, 17]. Using a notation as in $[3,9,17]$, we assume that a final time $T>0$ and some yield limit $r \geq 0$ are given and consider the play operator $\mathcal{P}_{r}[\cdot, \cdot]$ as operator from $\mathbb{R} \times \mathrm{C}[0, T]$ to $\mathrm{C}[0, T]$ mapping $\left(z_{0}, u\right) \in \mathbb{R} \times \mathrm{C}[0, T]$ (with $z_{0}$ being the initial state, $u$ being the input function and $\mathrm{C}[0, T]$ denoting the space of all continuous functions from $[0, T]$ to $\mathbb{R}$ ) to a function $\mathcal{P}_{r}\left[z_{0}, u\right] \in \mathrm{C}[0, T]$. For the function $\mathcal{P}_{r}\left[z_{0}, u\right]:[0, T] \rightarrow \mathbb{R}$, we denote by $\mathcal{P}_{r}\left[z_{0}, u\right](t)$ the value at $t$ for any $t \in[0, T]$. We recall that for $u \in \mathrm{C}[0, T]$ being piecewise monotone, i.e., such that there exists $0=t_{0}<t_{1}<\cdots<t_{n}=T$ with $u$ being monotone on $\left[t_{i-1}, t_{i}\right]$ for all $i \in\{1, \ldots, n\}$, it holds that $\mathcal{P}_{r}\left[z_{0}, u\right]$ is piecewise monotone and satisfies

$$
\begin{aligned}
& \mathcal{P}_{r}\left[z_{0}, u\right](0)=\max \left(u(0)-r, \min \left(u(0)+r, z_{0}\right)\right), \\
& \mathcal{P}_{r}\left[z_{0}, u\right](t)=\left\{\begin{array}{l}
\left.\max \left(\mathcal{P}_{r}\left[z_{0}, u\right]\left(t_{i-1}\right), u(t)-r\right)\right), \text { if } u \text { is increasing on }\left[t_{i-1}, t_{i}\right], \\
\left.\min \left(\mathcal{P}_{r}\left[z_{0}, u\right]\left(t_{i-1}\right), u(t)+r\right)\right), \text { if } u \text { is decreasing on }\left[t_{i-1}, t_{i}\right],
\end{array}\right.
\end{aligned}
$$

for all $\left.t \in] t_{i-1}, t_{i}\right]$ and all $i \in\{1, \ldots, n\}$. An example for an input function and the output of the play operator is shown in Figure 1. To define the function $\mathcal{P}_{r}\left[z_{0}, u\right]$ for general $u \in \mathrm{C}[0, T]$, a convergence argument is used, see, e.g., in [8].

In Figure 2, the outputs of the play for one input function and several yield limits are shown. Therein, we observe that even if $\mathcal{P}_{r}[0, u]$ and $\mathcal{P}_{r^{\prime}}[0, u]$ with $r \neq r^{\prime}$ coincide on some time interval, they may not coincide in the future.

In the next section, we need to deal with the evolutions of $\mathcal{P}_{r}[r, u]$ for all $r \geq 0$ simultaneously and are interested in an efficient method to evaluate for $t \in[0, T]$ the function $[0, \infty) \ni r \mapsto \mathcal{P}_{r}[r, u](t)$. The method in use is inspired by consideration for the Preisach operator and the representation of the Preisach operator by using the play operator and a memory sequence, allowing the evaluation according to Lemma 2.1.

Adapting the construction of the memory sequence as in ([9], Text from (II.2.11) up to Prop. II.2.5) to a play operator with an initial configuration being equal to some constant value $w \in \mathbb{R}$ on $[0,+\infty[$, we deal with piecewise monotone input functions $u \in \mathrm{C}[0, T]$, and $t \in[0, T]$, and consider the following construction: 

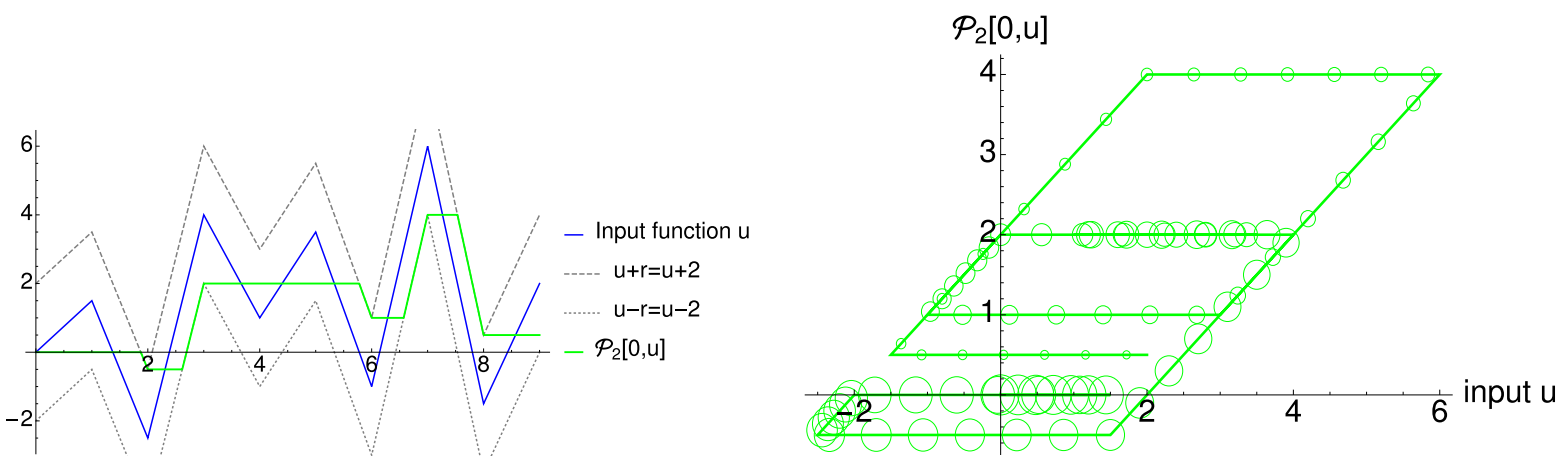

Figure 1. Left-hand side: evolution of input function $u$, of $u+2$, of $u-2$ and of $\mathcal{P}_{2}[0, u]$; right-hand side: input-output diagram, showing evolution of $\left(u(t), \mathcal{P}_{2}[0, u](t)\right)$, sizes of marking circles decreases with time.

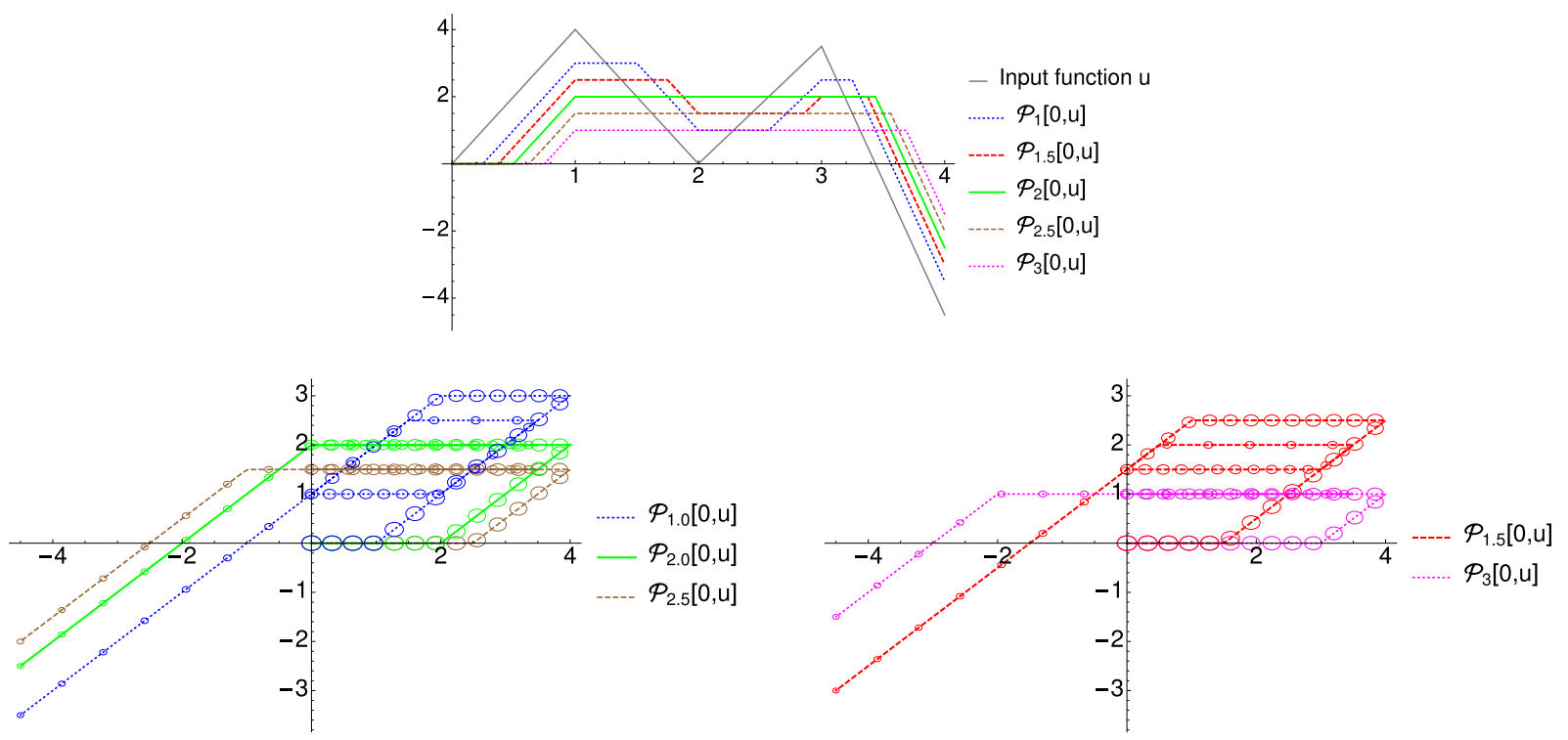

FIgURE 2. One input function and the output functions $\mathcal{P}_{r}[0, u]$ for the play-operator with yield limits $r \in\{1,1.5,2,2.5,3\}$. Top: evolution; bottom, left-hand side: input-output diagram for $r \in\{1,2,2.5\}$; bottom, right-hand side: input-output diagram for $r \in\{1.5,3\}$.

- Let

$$
\begin{aligned}
& r_{w, u, t, 0}:=\max \{|u(\tau)-w|: \tau \in[0, t]\} \\
& s_{w, u, t, 0}:=\max \left\{\tau \in[0, t]:|u(\tau)-w|=r_{w, u, t, 0}\right\} \\
& z_{w, u, t, 0}:=u\left(s_{w, u, t, 0}\right)
\end{aligned}
$$

- If $r_{w, u, t, 0}>0$ then it follows that $z_{w, u, t, 0} \neq w$. We define

$$
p_{w, u, t}:=\left\{\begin{array}{l}
1, \quad \text { if } \quad z_{w, u, t, 0}<w \\
-1, \quad \text { if } \quad z_{w, u, t, 0}>w .
\end{array}\right.
$$


For $k=1,2, \ldots$, we recursively define

$$
\begin{aligned}
z_{w, u, t, k} & := \begin{cases}\max \left\{u(\tau): \tau \in\left[s_{w, u, t, k-1}, t\right]\right\}, & \text { if } k \text { is odd and } p_{w, u, t}>0, \\
\max \left\{u(\tau): \tau \in\left[s_{w, u, t, k-1}, t\right]\right\}, & \text { if } k \text { is even and } p_{w, u, t}(t)<0, \\
\min \left\{u(\tau): \tau \in\left[s_{w, u, t, k-1}, t\right]\right\}, & \text { otherwise, }\end{cases} \\
r_{w, u, t, k}: & =\frac{1}{2}\left|z_{w, u, t, k}-z_{w, u, t, k-1}\right|, \\
s_{w, u, t, k}: & =\max \left\{\tau \in\left[s_{w, u, t, k-1}, t\right]: u(\tau)=z_{w, u, t, k}\right\},
\end{aligned}
$$

until it holds that $s_{w, u, t, k}(t)=t$. Then we define $K_{w, u, t}:=k$ and $r_{w, u, t, K_{w, u, t}+1}=0$. We have $s_{w, u, t, k}=t$ for some finite index $k$ since $u$ restricted to $[0, t]$ has different local extrema at $s_{w, u, t, 0}, s_{w, u, t, 1}, \ldots$, and the number of them is limited since $u$ is piecewise monotone. Moreover, we see that the construction yields that

$$
0=r_{w, u, t, K_{w, u, t}+1}<r_{w, u, t, K_{w, u, t}}<\cdots<r_{w, u, t, 1}<r_{w, u, t, 0}<\infty .
$$

Adapting now ([9], Prop. II.2.5), we get:

Lemma 2.1. In the considered situation, it holds:

a) $\mathcal{P}_{r}[w, u](t)=w$ for all $r \geq r_{w, u, t, 0}$.

b) If $r_{w, u, t, 0}>0$, we have

$$
\mathcal{P}_{r}[w, u](t)=z_{w, u, t, k}+(-1)^{k} p_{w, u, t} r, \quad \forall r \in\left[r_{w, u, t, k+1}, r_{w, u, t, k}\right], k \in\left\{0, \ldots, K_{w, u, t}\right\} .
$$

Proof.

a) Let $r \geq r_{w, u, t, 0}$ be given. Recalling (2.2a), we deduce that $u(\tau)-r \leq w \leq u(\tau)+r$ for all $\tau \in[0, t]$. In view of (2.1a), we conclude that $\mathcal{P}_{r}[w, u](0)=w$. Since $u$ is piecewise monotone, we can perform an induction argument over the monotonicity intervals and observe by using $(2.1 \mathrm{~b})$ that $\mathcal{P}_{r}[w, u](\tau)=w$ for all $\tau \in[0, t]$.

b) Assume that $r_{w, u, t, 0}>0$. Let $\lambda:[0, \infty) \rightarrow \mathbb{R}$ be defined by $\lambda(r):=0$ for all $r \geq 0$ if $w=0$; otherwise let

$$
\lambda(r):=\left\{\begin{array}{l}
w, \quad \text { if } \quad r \leq r_{w, u, t, 0}, \\
w\left(1-\frac{1}{|w|}\left(r-r_{w, u, t, 0}\right)\right), \quad \text { if } \quad r_{w, u, t, 0} \leq r \leq r_{w, u, t, 0}+|w|, \\
0, \quad \text { if } \quad r \geq r_{w, u, t, 0}+|w| .
\end{array}\right.
$$

For both definitions it holds that $\lambda \in W^{1, \infty}(0, \infty)$, that $\left|\frac{\mathrm{d} \lambda(r)}{\mathrm{d} r}\right| \leq 1$ for all $r \geq 0$ and that $\lambda(s)=0$ for all $s \geq r_{w, u, t, 0}+|w|$. This yield that $\lambda$ belongs to the subset $\Lambda_{0}$ of memory configurations as defined in II.(2.5) from [9].

Following II.(2.11) from [9], we define $m_{\lambda}: \mathbb{R} \rightarrow[0,+\infty[$ by

$$
m_{\lambda}(v):=\min \{r \geq 0:|v-\lambda(r)|=r\}, \quad \forall v \in \mathbb{R} .
$$

Then it follows that $m_{\lambda}(v)=|v-w|$ for all $v \in \mathbb{R}$ with $|v-w| \leq r_{w, u, t, 0}$. Recalling (2.2a), we see that this equation holds for $v=u(\tau)$ for all $\tau \in[0, t]$. Considering $\bar{r}$ and $\bar{t}$ as in II.(2.14) from [9], we deduce by recalling II.(2.12) from [9], (2.2a), (2.2b), and (2.3) that

$$
\begin{aligned}
\bar{r} & :=\max \left\{m_{\lambda}(u(\tau)): \tau \in[0, t]\right\} \\
& =\max \{|u(\tau)-w|: \tau \in[0, t]\}=r_{w, u, t, 0},
\end{aligned}
$$




$$
\begin{aligned}
\bar{t} & :=\max \left\{\tau \in[0, t]: m_{\lambda}(u(\tau))=\bar{r}\right\} \\
& =\max \left\{\tau \in[0, t]:|u(\tau)-w|=r_{w, u, t, 0}\right\}=s_{w, u, t, 0} \\
u(\bar{t})-\lambda(\bar{r}) & =u\left(s_{w, u, t, 0}\right)-w=-p_{w, u, t}\left|u\left(s_{w, u, t, 0}\right)-w\right| \\
& =-p_{w, u, t} r_{w, u, t, 0}=-p_{w, u, t} \bar{r}
\end{aligned}
$$

- If $p_{w, u, t}=1$ then it follows from (2.13) that $u(\bar{t})=\lambda(\bar{r})-\bar{r}$. We define $r_{k}:=r_{w, u, t, k}$ for all $k \in$ $\left\{0, \ldots, K_{w, u, t}+1\right\}$ and $t_{k}:=s_{w, u, t, k}$ for all $k \in\left\{0, \ldots, K_{w, u, t}\right\}$. Defining moreover $t_{K_{w, u, t}+1}=t, r_{k}:=0$ and $t_{k}:=t$ for all $k \in \mathbb{N}$ with $k>K_{w, u, t}+1$ and recalling the definition of a memory sequence as in the text before Proposition II.2.5 from [9], it follows that $\left\{\left(t_{j}, r_{j}\right)\right\}_{j=0}^{\infty}$ is the memory sequence of $u$ at the point $t$ with respect to the initial configuration $\lambda$. Moreover, it holds that $(-1)^{j}=p_{w, u, t}(-1)^{j}$ for all $j \in\{0,1,2, \ldots\}$.

- If $p_{w, u, t} \neq 1$ then it follows from (2.3) and (2.13) that $u(\bar{t})=\lambda(\bar{r})+\bar{r}$. We define $r_{k}:=r_{w, u, t, k-1}$ for all $k \in\left\{1, \ldots, K_{w, u, t}+2\right\}$ and $t_{k}:=s_{w, u, t, k-1}$ for all $k \in\left\{1, \ldots, K_{w, u, t}+1\right\}$. Defining moreover $t_{K_{w, u, t}+2}=t$, $r_{k}:=0$ and $t_{k}:=t$ for all $k \in \mathbb{N}$ with $k>K_{w, u, t}+2$ and recalling the definition of a memory sequence as in the text before Proposition II.2.5 from [9], it follows that $\left\{\left(t_{j}, r_{j}\right)\right\}_{j=1}^{\infty}$ is the memory sequence of $u$ at the point $t$ with respect to the initial configuration $\lambda$. Moreover, it holds that $(-1)^{j}=p_{w, u, t}(-1)^{j-1}$ for all $j \in\{0,1,2, \ldots\}$.

Recalling now II.(2.17) in Proposition II.2.5 from [9] and considering the value of $p_{w, u, t}$, we see that the assertion is proved.

\section{ForWARD UQ FOR THE PLAY OPERATOR WITH STOCHASTIC YIELD LIMIT}

Now, we want to consider a situation, wherein the true value of the yield limit is not known exactly, but we think that its value is near to 2. For this situation, we will do forward UQ to determine the influence of this uncertainty.

Hence, we interpret the yield limit $r \geq 0$ as value of the random variable $R$ generated from the normal distribution $N\left(2,0.5^{2}\right)$ with mean 2 and standard deviation 0.5 by ignoring $(-\infty, 0]$ and rescaling, leading to the following probability density function $\rho_{R}$ of $R$, see also Figure 3 :

$$
\rho_{R}(r)=\left\{\begin{array}{l}
\frac{1}{C} \mathrm{e}^{-\frac{(r-2)^{2}}{2 \cdot 0.5^{2}}}, \text { if } r \geq 0 \\
0, \quad \text { if } r<0,
\end{array} \quad \text { with } \quad C=\int_{0}^{\infty} \mathrm{e}^{-\frac{(r-2)^{2}}{2 \cdot 0.5^{2}}} \mathrm{~d} r\right.
$$

The mapping $[0, \infty) \ni r \mapsto \mathcal{P}_{r}[w, u](t)$ is continuous, see e.g., Corollary II.2.2 from [9]. Hence, it follows that the composition of this mapping with $R$ generates a random variable, denoted by $\mathcal{P}_{R}[w, u](t)$. The collection $\left(\mathcal{P}_{R}[w, u](t)\right)_{t \in[0, T]}$ is a stochastic process.

For an initial state $w \in \mathbb{R}$, an input function $u$ and a time $t \in[0, T]$, we denote by $\mu_{w, u, t}$ the probability measure on $\mathbb{R}$ for $\mathcal{P}_{R}[w, u](t)$ and observe that for the expected value $\mathbb{E}\left(\mathcal{P}_{R}[w, u](t)\right)$ it holds that

$$
\mathbb{E}\left(\mathcal{P}_{R}[w, u](t)\right)=\int_{0}^{\infty} \mathcal{P}_{r}[w, u](t) \rho_{R}(r) \mathrm{d} r=\int_{-\infty}^{\infty} s \mathrm{~d} \mu_{w, u, t}(s) .
$$

Hence, we see that the expected value is equal to the output of a Prandtl-Ishlinskiu operator with weight function $\rho_{R}$ and an initial state function that is equal to $w$ on $[0, \infty)$.

To compute $\mathcal{P}_{R}[w, u](t)$ or the measure $\mu_{w, u, t}$ by using $\left(\mathcal{P}_{r}[w, u](t)\right)_{r \geq 0}$, we consider the representation as in Lemma 2.1.

Let $r_{w, u, t, 0}$ be defined as in (2.2a). Then it follows from Lemma 2.1.a that $\mathcal{P}_{r}[w, u](t)=w$ for for all $r \geq$ $r_{w, u, t, 0}$. 


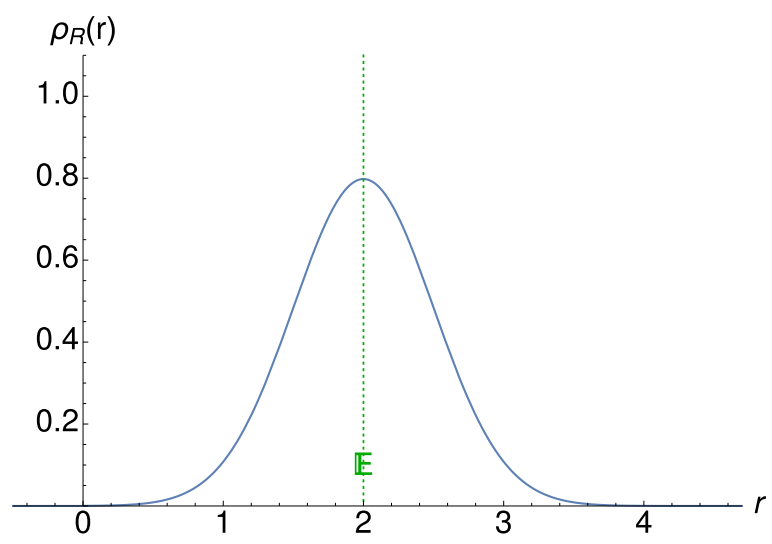

Figure 3 . Probability density $\rho_{R}$ and expected value $\mathbb{E}(R) \approx 2.00007$ of $R$ defined in $(3.1)$.

If $r_{w, u, t, 0}>0$, we consider $\left(r_{w, u, t, k}\right)_{k=1}^{K_{w, u, t}+1},\left(z_{w, u, t, k}\right)_{k=0}^{K_{w, u, t}}$ and $p_{w, u, t}$ as defined in (2.2c)-(2.5). Using Lemma 2.1.b, we see that $\left[r_{w, u, t, k+1}, r_{w, u, t, k}\right] \ni r \rightarrow \mathcal{P}_{r}[w, u](t) \in I_{w, u, t, k}$ with

$$
\begin{array}{r}
I_{w, u, t, k}:=\left[z_{w, u, t, k}+\min \left\{(-1)^{k} p_{w, u, t} r_{w, u, t, k},(-1)^{k} p_{w, u, t} r_{w, u, t, k+1}\right\},\right. \\
\left.z_{w, u, t, k}+\max \left\{(-1)^{k} p_{w, u, t} r_{w, u, t, k},(-1)^{k} p_{w, u, t} r_{w, u, t, k+1}\right\}\right]
\end{array}
$$

is a one-to-one mapping with the derivative $(-1)^{k} p_{w, u, t} \in\{1,-1\}$ such that it holds for $r \in\left[r_{w, u, t, k+1}, r_{w, u, t, k}\right]$ and $z \in I_{k}$ that $\mathcal{P}_{r}[w, u](t)=z$ if and only if $r=\frac{z-z_{w, u, t, k}}{(-1)^{k} p_{w, u, t}}$. Using this properties, we can show that the probability measure $\mu_{w, u, t}$ for the random variable $\mathcal{P}_{R}[0, u](t)$ is the sum of a Dirac measure at $w$ weighted by $\int_{r_{w, u, t, 0}} \rho_{R}(r) \mathrm{d} r=\int_{\max \{|u(\tau)-w|: \tau \in[0, t]\}} \rho_{R}(r) \mathrm{d} r$ and of a measure with a density on $\mathbb{R}$ being equal to 0 if $r_{w, u, t, 0}=0$ and being equal to

$$
\mathbb{R} \ni z \mapsto \sum_{i=0}^{K_{w, u, t}}\left\{\begin{array}{l}
0, \quad \text { if } z \notin I_{w, u, t, k}, \\
\rho_{R}\left(\frac{z-z_{w, u, t, k}}{(-1)^{k} p_{w, u, t}}\right) \text { if } \quad z \in I_{w, u, t, k},
\end{array}\right.
$$

otherwise.

As example, we consider the piecewise monotone input function $u \in \mathrm{C}[0, T]$ such that the value of the function increases linearly on $[0,1]$ from 0 to 4 , afterwards decreases linearly on $[1,2]$ to 0 , afterwards increases linearly on $[2,3]$ to 3.5 and afterwards decreases linearly on $[3,4]$ to -4.5 .

The probability measure $\mu_{0, u, t}$ for the random variable $\mathcal{P}_{R}[0, u](t)$ is the sum of a Dirac measure at 0 weighted by $\int_{\max \{|u(\tau)|: \tau \in[0, t]\}} \rho_{R}(r) \mathrm{d} r$ and of a measure with a density, denoted by $\phi_{t}$. For $t \in\left\{0,0.5,1,2,3,3 \frac{13}{32}, 4\right\}$, the values of $u(t)$, of the output $\mathcal{P}_{2}[0, u](t)$ of the play operator with yield limit 2 , of the expected value $\mathbb{E}\left(\mathcal{P}_{R}[0, u](t)\right)$ of $\left(\mathcal{P}_{R}[0, u](t)\right)$, of $\max \{|u(\tau)|: \tau \in[0, t]\}$ and of the weight $\int_{\max \{|u(\tau)|: \tau \in[0, t]\}} \rho_{R}(r) \mathrm{d} r$ are presented in Table 1 . Moreover, the density $\phi_{t}$, the expected value and the weight are also shown in Figures 4 and 5 .

To compute $\mathcal{P}_{r}[0, u]\left(\frac{13}{32}\right)$ as function of $r$ for $t=3 \frac{13}{32}=\frac{109}{32}$, we use Lemma 2.1. Using (2.2a)-(2.6) and the text afterwards, we get the values shown in Table 2. Hence, Lemma 2.1 yields that for $[0, \infty) \ni r \rightarrow \mathcal{P}_{r}[0, u]\left(\frac{13}{32}\right)$ it holds that the function increases linearly on $[0,1.625]$ from 0.25 to 1.875 , decreases linearly on $[1.625,1.75]$ to 1.75 , increases linearly on $[1.75,2]$ to 2 and decreases linearly on $[2,4]$ to 0 and is equal to 0 on $[4, \infty)$, see also Figure 6. For computing the corresponding density $\phi_{\frac{109}{32}}$, shown in the top, right-hand side of Figure 5 by using (3.4), we have to sum up several values for $\rho_{R}(r)$. In Figure 6 , the corresponding values are marked for $z \in\{0.1,1.3,1.8,1.9\}$. In view of the $I_{k}$ shown in Table 2 , we see that for $z \in[0,0.25)$ we need to evaluate $\rho_{R}(r)$ 
TABLE 1 . Values for the time $t$, the input function $u(t), \mathcal{P}_{2}[0, u](t), \mathbb{E}\left(\mathcal{P}_{R}[0, u](t)\right), \max \{|u(\tau)|$ : $\tau \in[0, t]\}$, and $\int_{\max \{|u(\tau)|: \tau \in[0, t]\}} \rho_{R}(r) \mathrm{d} r$; the values that have been chosen for the time $t$ are also considered in Figures 4 and 5.

\begin{tabular}{cccccc}
\hline$t$ & $u(t)$ & $\mathcal{P}_{2}[0, u](t)$ & $\mathbb{E}\left(\mathcal{P}_{R}[0, u](t)\right)$ & $\max _{\tau \in[0, t]}|u(\tau)|$ & $\int_{\max \{|u(\tau)|: \tau \in[0, t]\}} \phi_{R}(r) \mathrm{d} r$ \\
\hline 0 & 0 & 0 & 0 & 0 & 1 \\
0.5 & 2 & 0 & 0.199411 & 2 & 0.500016 \\
1 & 4 & 2 & 1.99994 & 4 & 0.0000316722 \\
2 & 0.5 & 2.5 & 1.60112 & 4 & 0.0000316722 \\
3 & 3.5 & 1.5 & 1.7988 & 4 & 0.0000316722 \\
$3 \frac{13}{32}$ & 0.25 & 2.25 & 1.66774 & 4 & 0.0000316722 \\
4 & -4.5 & -2.5 & -2.49993 & 4.5 & $2.8661 \cdot 10^{-7}$ \\
\hline
\end{tabular}
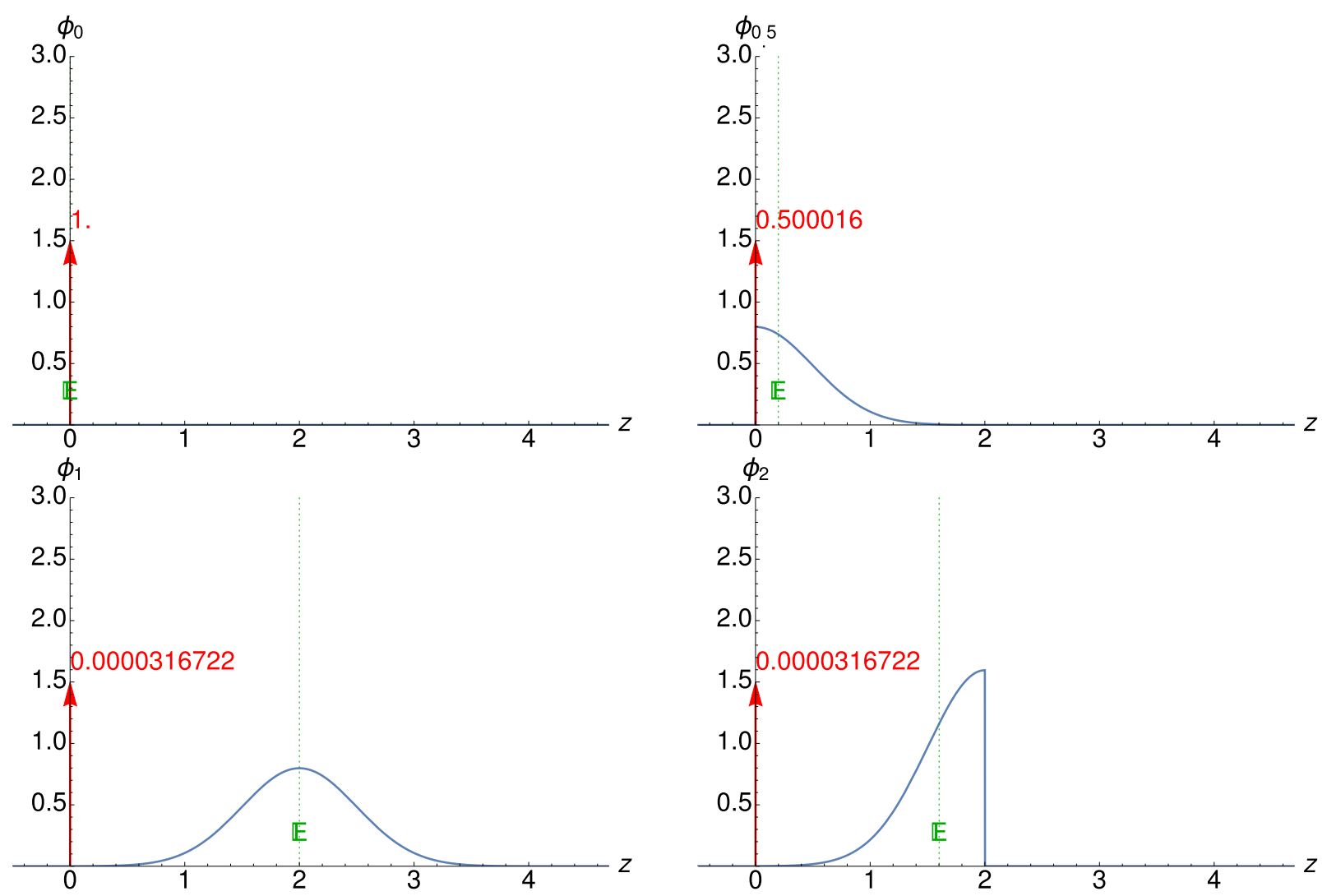

Figure 4. The density function $\phi_{t}$ and the weight $\int_{\max \{|u(\tau)|: \tau \in[0, t]\}} \phi_{R}(r) \mathrm{d} r$ for the Dirac measure concentrated at 0 leading to the probability measure $\mu_{0, u, t}$ of $\mathcal{P}_{R}[0, u](t)$ for some values of $t$, see also Table 1 . Also $\mathbb{E}\left(\mathcal{P}_{R}[0, u](t)\right)$ is shown, marked be $\mathbb{E}$. It holds top, left-hand side: $t=0$; top, right-hand side: $t=0.5 ;$ bottom, left-and side: $t=1 ;$ bottom, left-and side: $t=2$. 

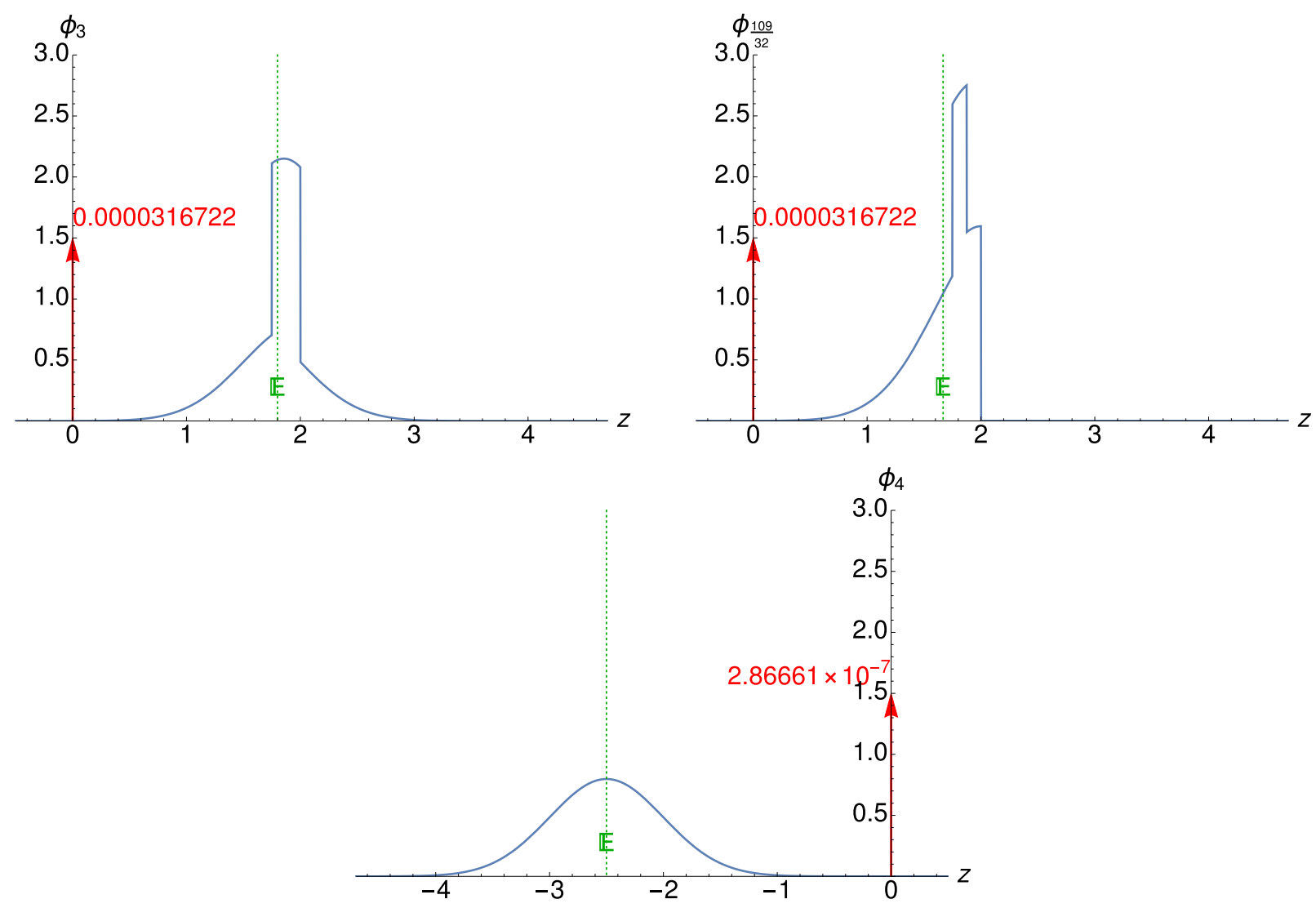

Figure 5. The density function $\phi_{t}$ and the weight $\int_{\max \{|u(\tau)|: \tau \in[0, t]\}} \phi_{R}(r) \mathrm{d} r$ for the Dirac measure concentrated at 0 leading to the probability measure $\mu_{0, u, t}$ of $\mathcal{P}_{R}[0, u](t)$ for some values of $t$, see also Table 1 . Also $\mathbb{E}\left(\mathcal{P}_{R}[0, u](t)\right)$ is shown, marked be $\mathbb{E}$. It holds top, left-hand side: $t=3$; top, right-hand side: $t=3 \frac{13}{32}=\frac{109}{32}$, bottom $t=4$.

TABLE 2. Values for $r_{0, u, 3 \frac{13}{32}, k}, s_{0, u, 3 \frac{13}{32}, k}, z_{0, u, 3 \frac{13}{32}, k}$ and the interval $I_{0, u, 3 \frac{13}{32}, k}$ for the considered input function. It holds $K_{0, u, 3 \frac{13}{32}}=3$ and $p_{0, u, 3 \frac{13}{32}}=-1$.

\begin{tabular}{ccccc}
\hline$k$ & $r_{0, u, 3 \frac{13}{32}, k}$ & $s_{0, u, 3 \frac{13}{32}, k}$ & $z_{0, u, 3 \frac{13}{32}, k}$ & $I_{0, u, 3 \frac{13}{32}, k}$ \\
\hline 0 & 4 & 1 & 4 & {$[0,2]$} \\
1 & 2 & 2 & 0 & {$[1.75,2]$} \\
2 & 1.75 & 3 & 3.5 & {$[1.75,1.875]$} \\
3 & 1.625 & $3 \frac{13}{32}$ & 0.25 & {$[0.25,1.875]$} \\
4 & 0 & - & - & \\
\hline
\end{tabular}

for one value of $r$ to evaluate (3.4). In the corresponding computation for $z \in[0.25,1.75) \cup(1.875,2]$, we have to sum up two values of $\rho_{R}$, while for $z \in[1.75,1.875]$ we have to sum up four values.

As one can see in Figure 7 , showing evolutions of $\mathcal{P}_{2}[0, u](t)$ and of the expected value $\mathbb{E}\left(\mathcal{P}_{R}[0, u](t)\right)$, it holds that these evolutions differ. Since Corollary II.2.2 from [9] yields that

$$
\left|\mathcal{P}_{2}[0, u](t)-\mathcal{P}_{\mathbb{E}(R)}[0, u](t)\right| \leq|\mathbb{E}(R)-2|=0.00007, \quad \forall t \in[0, T],
$$




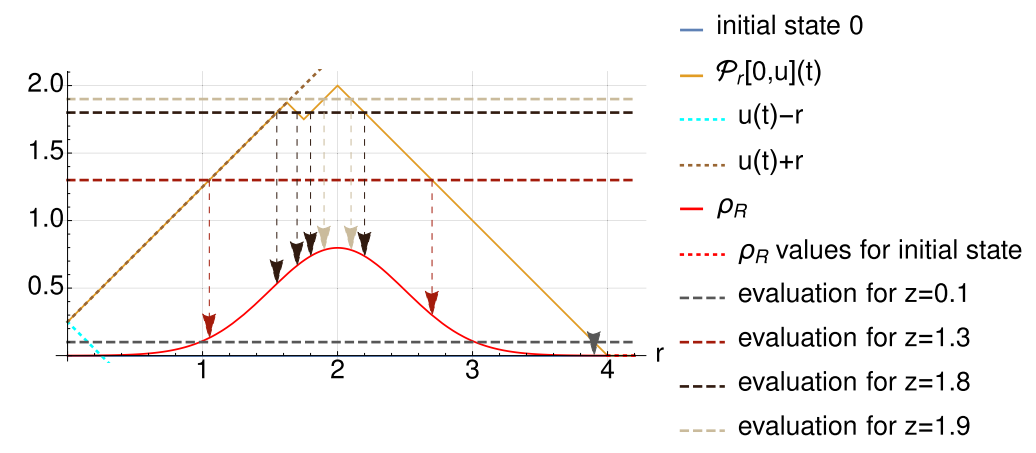

FiguRE 6. Output of play operator $\mathcal{P}_{r}[0, u](t)$ as function of $r$ for $t=3 \frac{13}{32}=\frac{109}{32}$ and evaluating value for $\rho_{R}$ during computing the sum as in $(3.4)$ for $z \in\{0.1,1.3,1.8,1.9\}$.

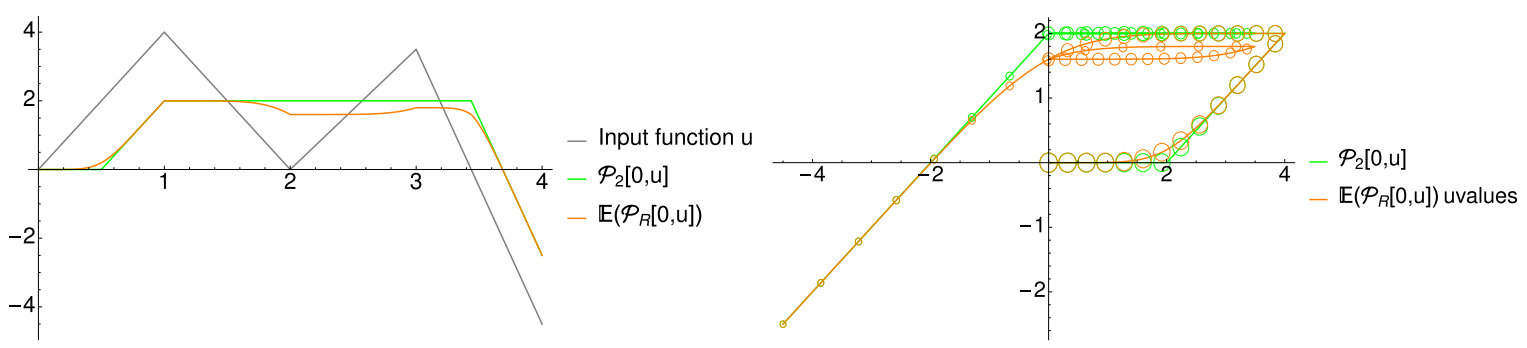

Figure 7. Left-hand side: evolution of $\mathcal{P}_{2}[0, u](t)$ (indistinguishable from evolution of $\mathcal{P}_{\mathbb{E}(R)}[0, u](t)$ in the considered scale) and of the expected value $\mathbb{E}\left(\mathcal{P}_{R}[0, u](t)\right)$; right-hand side: corresponding input-output diagram.

such that in the scale used in this figure the graphs of $\mathcal{P}_{2}[0, u]$ and $\mathcal{P}_{\mathbb{E}(R)}[0, u]$ would coincide, we deduce that also the evolutions of $\mathcal{P}_{\mathbb{E}(R)}[0, u](t)$ and of the expected value $\mathbb{E}\left(\mathcal{P}_{R}[0, u](t)\right)$ differ.

\section{Generalized Prandth-Ishlinskil operators}

Following [6, 7, 18], we consider generalized Prandtl-Ishlinskǐ operators that are defined by combining a Prandtl-Ishlinskil operators and a given function $g: \mathbb{R} \rightarrow \mathbb{R}$ by mapping an input function $u:[0, T] \rightarrow[0, T]$ to the output of the Prandtl-Ishlinskil considered with the composition $g \circ u$ used as input function. These kind of operators can be inverted analytically and can model effects like saturation, asymptotic behaviors, etc., see, e.g., discussions in $[2,5,18]$.

In Sections 3 and 5 from [4] the following generalized Prandtl-Ishlinskiur operator has been used to model the magnetization of Galfenol for an applied magnetic field $H$. This operator is defined by mapping $H \in \mathrm{C}[0, T]$ to $\mathcal{G}_{c_{1}, c_{2}, c_{3}}[H] \in \mathrm{C}[0, T]$ defined by

$$
\mathcal{G}_{c_{1}, c_{2}, c_{3}}[H](t)=\mathcal{P} \mathcal{I}_{c_{1}, c_{2}}\left[\tanh \left(c_{3} H\right)\right](t), \quad \forall t \in[0, T],
$$

with the Prandtl-Ishlinskil operator $\mathcal{P} \mathcal{I}_{c_{1}, c_{2}}: \mathrm{C}[0, T] \rightarrow \mathrm{C}[0, T]$ being defined by

$$
\left.\mathcal{P I}_{c_{1}, c_{2}}[u)\right](t)=\int_{0}^{\infty} c_{1} \mathrm{e}^{-r / c_{2}} \mathcal{P}_{r}\left[\lambda_{0}(r), u\right](t) \mathrm{d} r, \quad \forall u \in \mathrm{C}[0, T], t \in[0, T],
$$

with $c_{1}, c_{2}, c_{3}$ being positive parameters and $\lambda_{0}:[0, \infty) \rightarrow \mathbb{R}$ being an appropriate function. 
Related to a Prandtl-Ishlinskiur operator is the initial loading curve, whose value at $x \in \mathbb{R}$ can be determined by letting the input monotonically increase or decrease linearly from 0 to $x$ on $[0, T]$ with an initial configuration $\lambda$ being constant 0 , and evaluating the output of the Prandtl-Ishlinskiur operator at $T$.

In applications to plasticity modeled by a Prandtl-Ishlinskiur operator this curve can be measured by considering a material with no previous memory, e.g., if the memory was erased for instance by heating above the Curie temperature and cooling again or applying an input function with decreasing oscillations.

The initial loading curve $\psi_{c_{1}, c_{2}}$ for $\mathcal{P} \mathcal{I}_{c_{1}, c_{2}}$ satisfies

$$
\begin{aligned}
\psi_{c_{1}, c_{2}}(s) & =\int_{0}^{\infty} c_{1} \mathrm{e}^{-r / c_{2}}\left\{\begin{array}{l}
(s-r), \quad \text { if } r \leq s, \\
0, \quad \text { if }-r \leq s \leq r, \quad \mathrm{~d} r \\
(s+r), \quad \text { if } r \geq-s,
\end{array}\right. \\
& =s c_{1} c_{2}+c_{1} c_{2}^{2}\left\{\begin{array}{l}
\mathrm{e}^{-\frac{s}{c_{2}}}-1, \quad \text { if } \quad s \geq 0, \\
1-\mathrm{e}^{\frac{s}{c_{2}}}, \quad \text { if } \quad s \leq 0,
\end{array}\right.
\end{aligned}
$$

for all $s \in \mathbb{R}$.

Following, $[5,6,12]$, we consider $u \in C[0, T]$ and $0 \leq t_{a}<t_{b}<t_{c} \leq T$ with $u$ being monotone on $\left[t_{a}, t_{b}\right]$ and on $\left[t_{b}, t_{c}\right]$ such that $u\left(t_{a}\right)=u\left(t_{c}\right)$. Recalling $(2.1 \mathrm{~b})$, we get for all $w_{0} \in \mathbb{R}$ that

$$
\mathcal{P}_{r}\left[w_{0}, u\right]\left(t_{c}\right)-\mathcal{P}_{r}\left[w_{0}, u\right]\left(t_{b}\right)=2\left\{\begin{array}{l}
0, \quad \text { if }\left|\frac{u\left(t_{c}\right)-u\left(t_{b}\right)}{2}\right| \leq r, \\
\frac{u\left(t_{c}\right)-u\left(t_{b}\right)}{2}-r, \quad \text { if } \frac{u\left(t_{c}\right)-u\left(t_{b}\right)}{2}>r, \\
\frac{u\left(t_{c}\right)-u\left(t_{b}\right)}{2}+r, \quad \text { if }-\frac{u\left(t_{c}\right)-u\left(t_{b}\right)}{2}>r .
\end{array}\right.
$$

Therefore, invoking (4.3) and (4.2), we get

$$
\begin{aligned}
& \psi_{c_{1}, c_{1}}\left(\left|\frac{u\left(t_{c}\right)-u\left(t_{b}\right)}{2}\right|\right) \\
= & \frac{1}{2}\left\{\begin{array}{lll}
\mathcal{P} \mathcal{I}_{c_{1}, c_{2}}\left[\lambda_{0}, u\right]\left(t_{c}\right)-\mathcal{P} \mathcal{I}_{c_{1}, c_{2}}\left[\lambda_{0}, u\right]\left(t_{b}\right), & \text { if } \quad u\left(t_{c}\right) \geq u\left(t_{b}\right), \\
\mathcal{P I}_{c_{1}, c_{2}}\left[\lambda_{0}, u\right]\left(t_{b}\right)-\mathcal{P} \mathcal{I}_{c_{1}, c_{2}}\left[\lambda_{0}, u\right]\left(t_{c}\right), & \text { if } \quad u\left(t_{b}\right) \leq u\left(t_{c}\right) .
\end{array}\right.
\end{aligned}
$$

Considering an input cycle and differences for corresponding measurements for a process that one would like to represent by a Prandtl-Ishlinskiu operator, one can derive an approximation for the initial loading curve on $\left[0,\left|\left(u\left(t_{2}\right)-u\left(t_{b}\right)\right) / 2\right|\right]$ from the measurements during $\left[t_{1}, t_{2}\right]$ by replacing on the right-hand side of $(4.5)$ the values of the operator by corresponding values from the measurements.

\section{IDENTIFICATION AND INVERSE UQ}

\subsection{Considered situation}

In [1], a magnetostrictive Terfenol-actuator is investigated and the hysteresis between the reference signal determining the current generating the magnetic field and the resulting displacement is considered, and the data of the First-Order-Reversal-Curves (FORCs) are used to determine a Preisach operator and a generalized Prandtl-Ishlinskil̆ operator.

We will now use the data measured during the preparation of the FORC-diagram in [1], considering now the measured current (corrente) generated from the reference signal as input data, such that we get Figure 8 from the measurements, quite similar to the FORC diagram in Figure 4a from [1], except for the scale of the input.

Now, we will approximate these data by the initial loading curve corresponding to $\mathcal{G}_{c_{1}, c_{2}, c_{3}}[H](t)$, i.e., we consider the differences derived from the function $\tanh \left(c_{3} I(t)\right)$ as input for $\psi_{c_{1}, c_{1}}$. This will be used to determine 


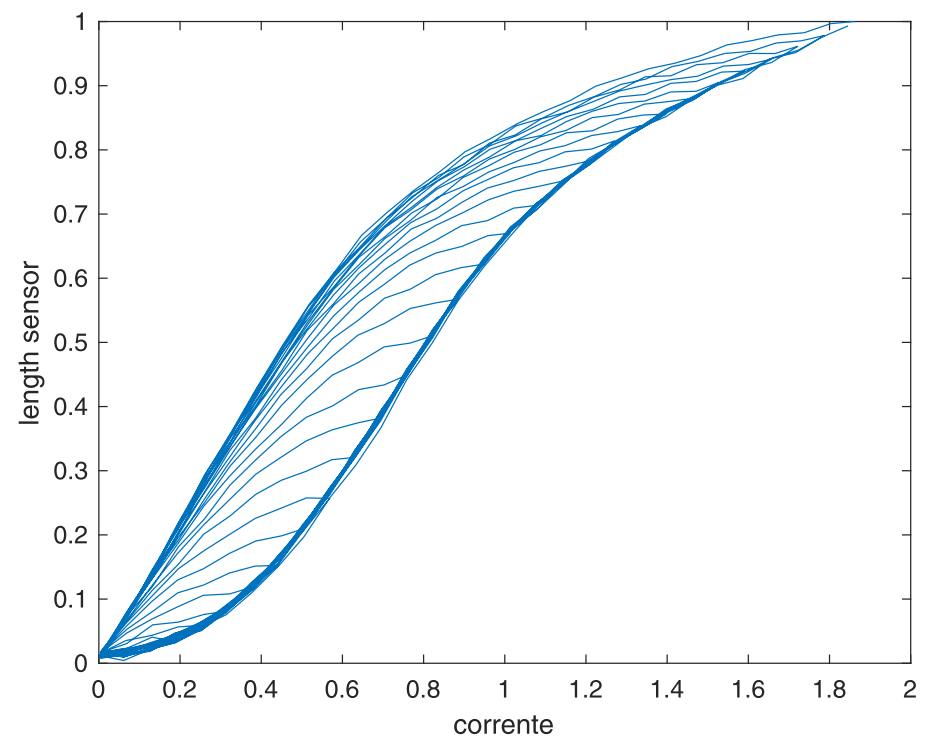

FiguRE 8. FORCs and further measurements during creation of these curves.

an appropriate value for $c_{3}$ and to derive information about $c_{1}$ and $c_{2}$ and their uncertainty, i.e., for these parameters we will perform inverse UQ.

\subsection{Identification of $c_{3}$}

To determine $c_{3}$, we consider the generated approximations of the initial loading curve. For $i \in\{30, \cdots, 58\}$, we have sets of times $t_{i, 0}<t_{i, 1}<\cdots<t_{i, K_{i}}$ such that the current $I$ as function of time has a discrete local maximum at $t_{i, 0}$ and it holds $I\left(t_{i, 0}\right)>I\left(t_{i, 1}\right)>\cdots>I\left(t_{i, K_{i}}\right)$. For $i \in\{1, \cdots, 29\}$ it holds that the current $I$ has a discrete local minima at $t_{i, 0}$ and it holds $I\left(t_{i, 0}\right)<I\left(t_{i, 1}\right)<\cdots<I\left(t_{i, K_{i}}\right)$.

Let $L$ denote the relative length change measured by the length sensor as function of time. Then it follows that the data sets $\left(I\left(t_{i, k}\right), L\left(t_{i, k}\right)\right)_{k=0}^{K_{i}}$ for $i=30, \ldots, 58$ represent the FORCs.

To derive the initial loading curve $\zeta_{i, c_{3}}$ for $i \in\{1,2, \ldots, 58\}$ and for $c_{3}>0$ by considering the data set $\left(I\left(t_{i, k}\right), L\left(t_{i, k}\right)\right)_{k=0}^{K_{i}}$ and the transformation $x \mapsto \tanh \left(c_{3} x\right)$, we consider (4.5) with $\psi_{c_{1}, c_{1}}$ replaced by $\zeta_{i, c_{3}}$, $\mathcal{P} \mathcal{I}_{c_{1}, c_{2}}\left[\lambda_{0}, u\right]$ replaced by $L, u(t):=\tanh \left(c_{3} I(t)\right), t_{b}=t_{i, 0}$, and $t_{c}=t_{i, k}$ for all $k=0, \ldots, K_{i}$, leading to

$$
\begin{aligned}
& \zeta_{i, c_{3}}\left(\frac{\left|\tanh \left(c_{3} I\left(t_{i, k}\right)\right)-\tanh \left(c_{3} I\left(t_{i, 0}\right)\right)\right|}{2}\right) \\
= & \frac{1}{2}\left\{\begin{array}{ll}
L\left(t_{i, k}\right)-L\left(t_{i, 0}\right), & \text { if } i \leq 29, \\
L\left(t_{i, 0}\right)-L\left(t_{i, k}\right), & \text { if } \quad i>29,
\end{array} \quad \forall k \in\left\{0,1, \ldots, K_{i}\right\}, i \in\{1, \ldots, 58\} .\right.
\end{aligned}
$$

By interpolation, we get a function $\zeta_{i, c_{3}}$ defined on $\left[0, \frac{1}{2}\left|\tanh \left(c_{3} I\left(t_{i, k}\right)\right)-\tanh \left(c_{3} I\left(t_{i, 0}\right)\right)\right|\right]$.

Now, $c_{3}$ is determined by requesting that the sum over the squared $L^{2}$-difference between these generated approximations is minimized. We end up with the optimal value $c_{3}=c_{\tanh }=0.682138$. The corresponding $\left(\zeta_{i, c_{\text {tanh }}}\right)_{i=1}^{58}$ set of initial loading curves is shown in Figure 9.

\subsection{Identification of $c_{1, i}$ and $c_{2, i}$}

For each approximation $\zeta_{i, c_{\mathrm{tanh}}}$ of the initial loading curve derived from the measurements by using interpolation, we determine $\left(c_{1, i}, c_{2, i}\right) \in(0,250) \times(0.0000001,25)$ by minimizing the $L^{2}$-difference between 


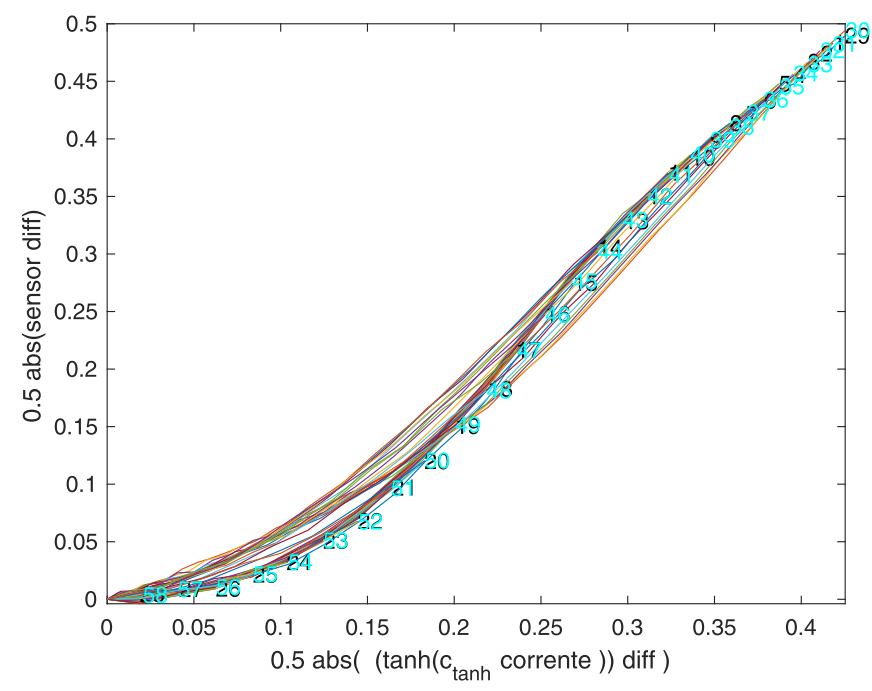

FIGURE 9. Derived initial loading curves.

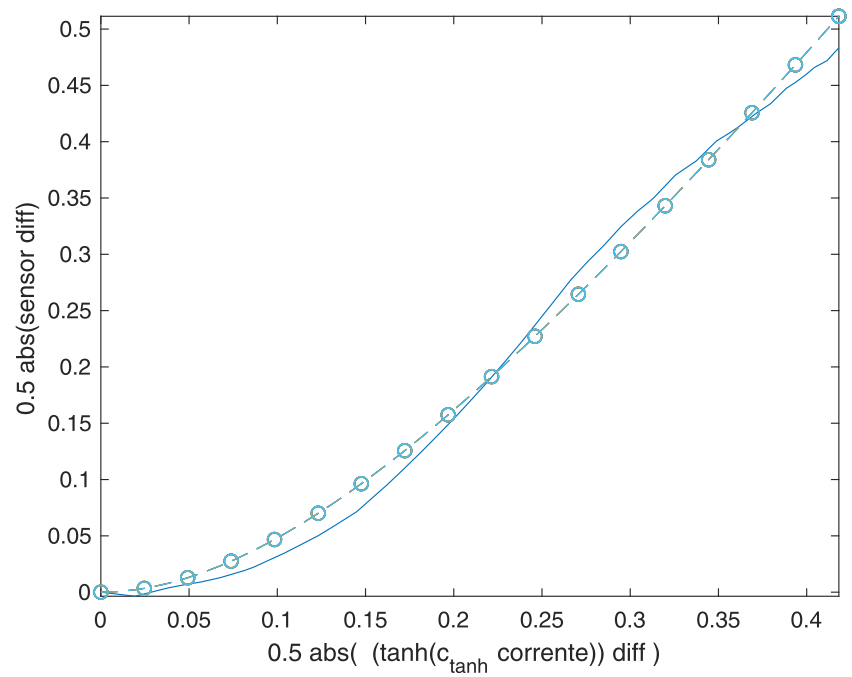

FIgURE 10. The first measured initial loading curve (solid line) and $\psi_{c_{1, i}, c_{2, i}}$ with $i=1$ (dashed line).

this approximation for the initial loading curve and the $\psi_{c_{1, i}, c_{2, i}}$ initial loading curve corresponding to the Prandtl-Ishlinskiı-operator $\mathcal{P \mathcal { I }}_{c_{1, i}, c_{2, i}}$, see an example in Figure 10.

Moreover, a data pair $\left(\overline{c_{1}}, \overline{c_{2}}\right)$ has been computed by minimizing the sum over the $L^{2}$-difference between $\zeta_{i, c_{\text {tanh }}}$ and $\psi_{\overline{c_{1}}, \overline{c_{2}}}$ for all $i \in\{1, \ldots, 58\}$, leading to $\overline{c_{1}}=11.7463$ and $\overline{c_{2}}=0.1636$.

If only the computed parameter pairs for the curves generated for decreasing inputs i.e., the pairs belonging to the FORCs, are considered, and also the pairs belonging to the three shortest definition intervals are ignored, we get the appropriate subset $\left(\left(c_{1, i}, c_{2, i}\right)\right)_{i=30}^{55}$ of 26 parameter pairs. Computing for this subset the mean and the standard deviation of the corresponding values for $c_{1, i}$ and $c_{2, i}$ leads to mean ${ }_{i}\left(c_{1, i}\right)=13.3218, \operatorname{mean}_{i}\left(c_{2, i}\right)=$ $0.116617, \operatorname{std}\left(c_{1, i}\right)=2.67912$, and $\operatorname{std}\left(c_{2, i}\right)=0.043755$. 

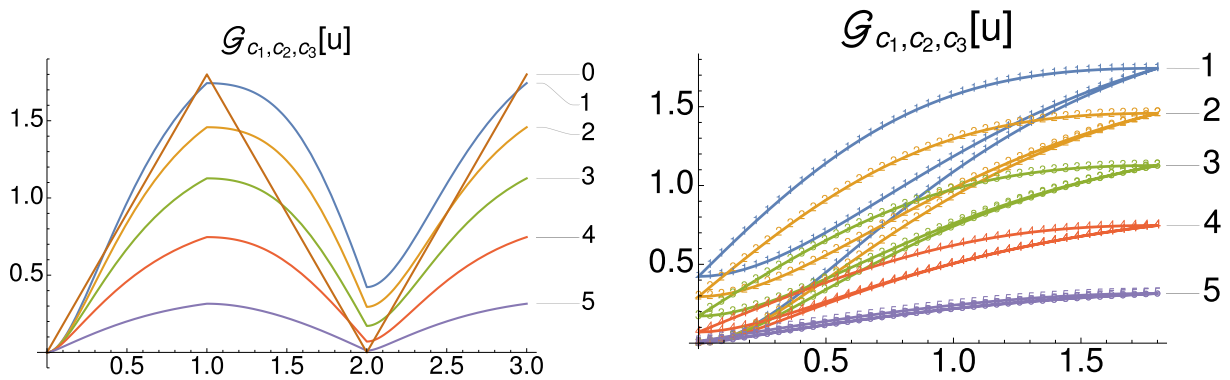

FIgURE 11. Input-output plot for $\mathcal{P} \mathcal{I}_{\operatorname{mean}_{i}\left(c_{1, i}\right), \operatorname{mean}_{i}\left(c_{2, i}\right), c_{3}}[u]$, $\mathcal{P} \mathcal{I}_{\text {mean }_{i}\left(c_{1, i}\right), \operatorname{mean}_{i}\left(c_{2, i}\right) \pm \operatorname{std}_{i}\left(c_{2, i}\right), c_{3}}[u]$, and $\mathcal{P} \mathcal{I}_{\text {mean }_{i}\left(c_{1, i}\right), \operatorname{mean}_{i}\left(c_{2, i}\right) \pm 2 \operatorname{std}_{i}\left(c_{2, i}\right), c_{3}}[u]$, for the initial state $\lambda_{0} \equiv 0$ and an input function $u$ increasing linearly from 0 to 1.8 , decreasing linearly afterwards to 0 and increasing linearly to 1.8 afterwards, Left-hand side: evolution of 0 - input $u, 1-\mathcal{P} \mathcal{I}_{\operatorname{mean}_{i}\left(c_{1, i}\right), \operatorname{mean}_{i}\left(c_{2, i}\right)-2 \operatorname{std}_{i}\left(c_{2, i}\right), c_{3}}[u], 2-\mathcal{P} \mathcal{I}_{\operatorname{mean}_{i}\left(c_{1, i}\right), \operatorname{mean}_{i}\left(c_{2, i}\right)-\operatorname{std}_{i}\left(c_{2, i}\right), c_{3}}[u]$, $3-\mathcal{P} \mathcal{I}_{\text {mean }_{i}\left(c_{1, i}\right), \operatorname{mean}_{i}\left(c_{2, i}\right), c_{3}}[u], \quad 4-\mathcal{P} \mathcal{I}_{\operatorname{mean}_{i}\left(c_{1, i}\right), \operatorname{mean}_{i}\left(c_{2, i}\right)+\operatorname{std}_{i}\left(c_{2, i}\right), c_{3}}[u], \quad 5 \quad-$ $\mathcal{P} \mathcal{I}_{\text {mean }_{i}\left(c_{1, i}\right), \operatorname{mean}_{i}\left(c_{2, i}\right)+2 \operatorname{std}_{i}\left(c_{2, i}\right), c_{3}}[u]$, right-hand side: input-output plots for $1-5$.

One could now use this data to start further investigations. As simple example, we consider $\mathcal{P} \mathcal{I}_{\text {mean }_{i}\left(c_{1, i}\right), \operatorname{mean}_{i}\left(c_{2, i}\right), c_{3}}[u], \mathcal{P} \mathcal{I}_{\text {mean }_{i}\left(c_{1, i}\right), \operatorname{mean}_{i}\left(c_{2, i}\right) \pm \operatorname{std}_{i}\left(c_{2, i}\right), c_{3}}[u]$, and $\mathcal{P} \mathcal{I}_{\operatorname{mean}_{i}\left(c_{1, i}\right), \operatorname{mean}_{i}\left(c_{2, i}\right) \pm 2 \operatorname{std}_{i}\left(c_{2, i}\right), c_{3}}[u]$, for the initial state $\lambda_{0} \equiv 0$ and an input function $u$ increasing linearly from 0 to 1.8 , decreasing afterwards linearly to 0 and increasing linearly to 1.8 leading to the outputs shown in Figure 11.

As an example for forward UQ, we now assume that the value of $c_{2}$ can be represented by a random variable $C_{2}$ with with density $\rho_{C_{2}}$ that is derived from the distribution $N\left(\operatorname{mean}_{i}\left(c_{2, i}\right),\left(\operatorname{std}_{i}\left(c_{2, i}\right)\right)^{2}\right)=$ $N\left(0.116617,(0.043755)^{2}\right)$ by ignoring $\left.]-\infty, 0.0000001\right]$ and rescaling, see the left-hand side of Figure 12 . In the right-hand side of this figure, an input function $u$, the graph of $\mathcal{P} \mathcal{I}_{\operatorname{mean}_{i}\left(c_{1, i}\right), \operatorname{mean}_{i}\left(c_{2, i}\right)}[u]$ (graph output does not change in this scale, if $\operatorname{mean}_{i}\left(c_{2, i}\right)=0.116617$ is replaced by $\left.\mathbb{E}\left(C_{2}\right)=0.11712\right)$ and the graph of $[0, T] \ni t \mapsto \mathbb{E}\left(\mathcal{P I}_{\text {mean }_{i}\left(c_{1, i}\right), C_{2}}[u](t)\right)$ with $\lambda_{0} \equiv 0$ are shown. In this example there is only a small difference between these graphs.

If all computed parameter pairs are considered, computing the mean and the standard deviation for all values for $c_{1}$ and $c_{2}$, we get $\operatorname{mean}_{i, \text { all }}\left(c_{1, i}\right)=16.0156, \operatorname{mean}_{i, \text { all }}\left(c_{2, i}\right)=4.71742, \operatorname{std}_{i, \text { all }}\left(c_{1, i}\right)=13.3218$, and $\operatorname{std}_{i, \text { all }}\left(c_{2, i}\right)=7.94603$. In view of these values, it is obvious, that one can not use them to derive a satisfying assumption for the random variables representing the positive parameters $c_{1}$ and $c_{2}$.

We could consider $N\left(m_{1}, \delta_{1}^{2}\right)$ and $N\left(m_{2}, \delta_{2}^{2}\right)$ for some positive constants $m_{1}, m_{2}, \delta_{1}, \delta_{2}$ and could perform some truncation, to derive random variables $C_{1}$ and $C_{2}$ to be used in UQ to represent an appropriate approximation for $c_{1}$ and $c_{2}$. But, this could only be justified if the pairs $\left(\left(c_{1, i}, c_{2, i}\right)\right)_{i=1}^{58}$ or the pairs $\left(\left(c_{1, i}, c_{2, i}\right)\right)_{i=30}^{55}$ could be considered as typical independent samples for $N\left(m_{1}, \delta_{1}^{2}\right)$ and $N\left(m_{2}, \delta_{2}^{2}\right)$. A typical example for the cloud generated by this kind of samples is shown in Figure 13 for $\left(N\left(1,1^{2}\right), N\left(2,\left(\frac{1}{2}\right)^{2}\right)\right)$.

Considering the computed parameter pairs $\left(\left(c_{1, i}, c_{2, i}\right)\right)_{i=1}^{58}$, see Figure 14, it is obvious that we can not get these set of pairs as samples if we assume that the parameters can be represented by two independent normal distributed random variables, even if the correlation between $\left(c_{1, i}\right)_{i}$ and $\left(c_{2, i}\right)_{i}$ is only -0.0737148 . Also the 26 data pairs $\left(\left(c_{1, i}, c_{2, i}\right)\right)_{i=30}^{55}$ in the appropriate subset introduced in Section 5.3, see Figure 15, with a corresponding correlation of -0.532375 , can not be derived as samples in this way.

Hence, we are interested in finding a more general random variable $\left(C_{1}^{*}, C_{2}^{*}\right)$ with values in $(0, \infty) \times(0, \infty)$ such that either $\left(\left(c_{1, i}, c_{2, i}\right)\right)_{i=1}^{58}$ or $\left(\left(c_{1, i}, c_{2, i}\right)\right)_{i=30}^{55}$ could be considered as typical samples of this random variable. 

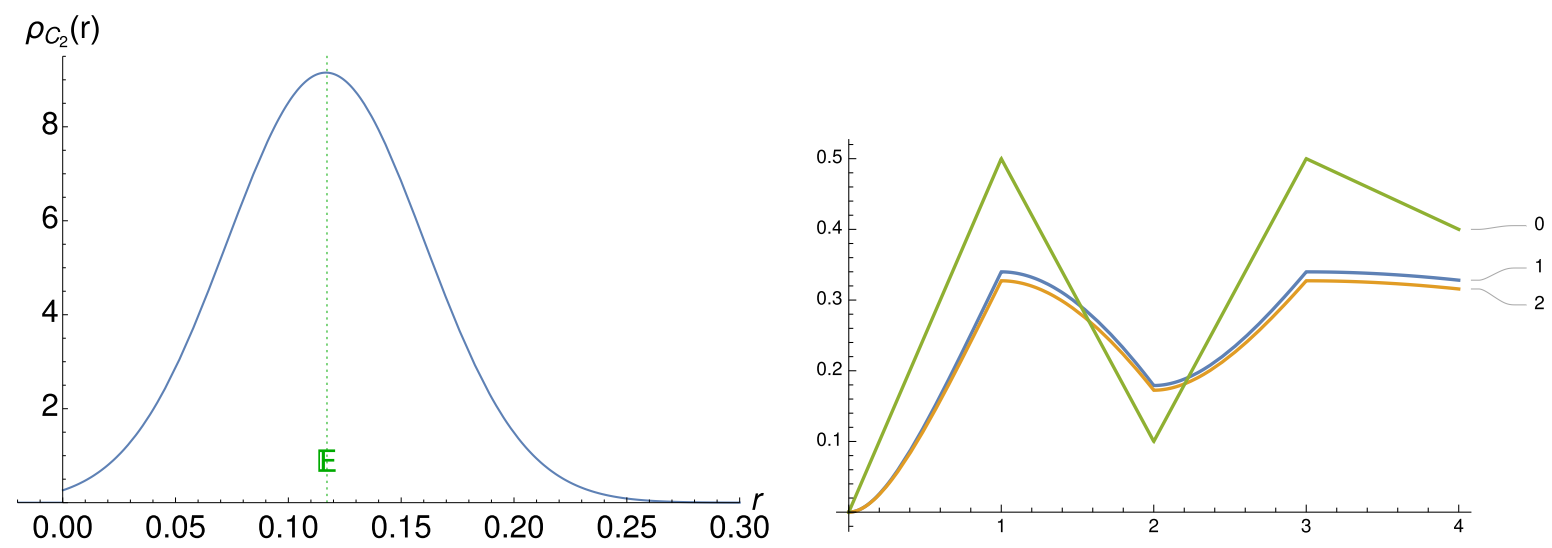

Figure 12. Left-hand side: Density $\rho_{2}$ of random variable $C_{2}$ describing $c_{2}$ derived from $N\left(\operatorname{mean}_{i}\left(c_{1,2}\right),\left(\operatorname{std}_{i}\left(c_{2,2}\right)\right)^{2}\right)=N\left(0.116617,(0.043755)^{2}\right)$ by ignoring $(-\infty, 0.0000001]$ and rescaling, expected value $\mathbb{E}\left(C_{2}\right)=0.11712$ is marked by $\mathbb{E}$. Right-hand side: an Input function $u$ (marked by 0 ), graph of $\mathcal{P} \mathcal{I}_{\text {mean }_{i}\left(c_{1}\right), \operatorname{mean}_{i}\left(c_{2}\right)}[u]$ (marked by 1 ) and graph of $\mathbb{E}\left(\mathcal{P} \mathcal{I}_{\text {mean }_{i}\left(c_{1, i}\right), C_{2}}[u]\right)$ (marked by 2$)$.

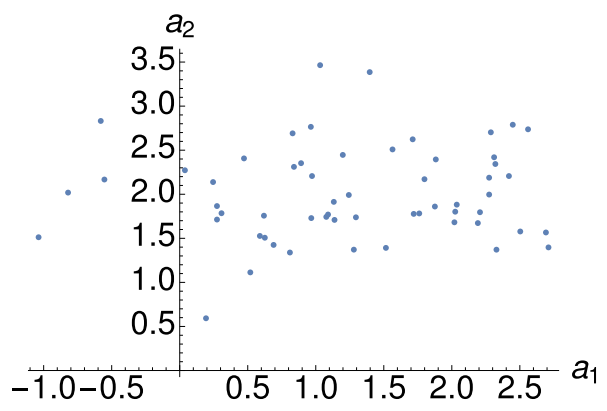

Figure 13. 58 independent samples for $\left(A_{1}, A_{2}\right)=\left(N\left(1,(1)^{2}\right), N\left(2,\left(\frac{1}{2}\right)^{2}\right)\right)$.
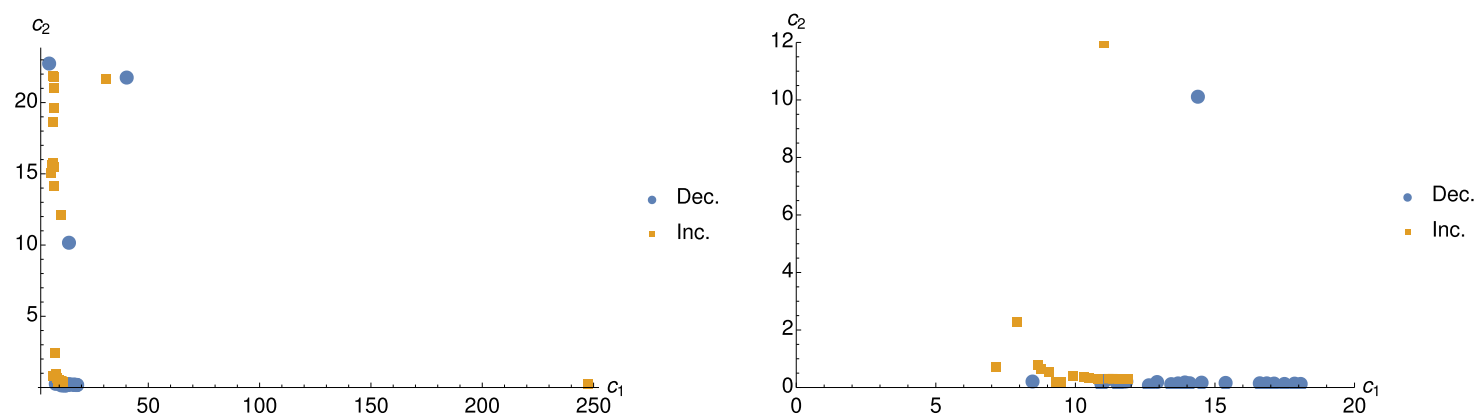

FIGURE 14. Sample pairs $\left(c_{1,1}, c_{2,1}\right), \cdots,\left(c_{1,58}, c_{2,58}\right)$. Left-hand side: plot of all pairs, righthand side: zoom plot. 


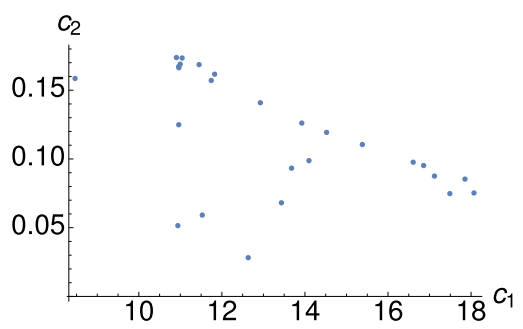

FIgURE 15. Appropriate subset $\left(c_{1,30}, c_{2,30}\right), \cdots,\left(c_{1,55}, c_{2,55}\right)$ of 26 parameter pairs introduced in Section 5.3.

\subsection{Applications of Bayes' Theorem}

Now, we will use Bayes' Theorem to derive a probability density on $(0, \infty) \times(0, \infty)$ by considering the measured data, aiming to generate $C_{1}^{*}, C_{2}^{*}$ as discussed above.

Let $v^{\text {obs }}$ be a vector containing the sets of the non-trivial discrete values for the initial loading curve used to derived their approximations in Sections 5.2 and 5.3, i.e.,

$$
v^{\text {obs }}=\left(\begin{array}{ll}
\left(\frac{1}{2}\left(L\left(t_{i, k}\right)-L\left(t_{i, 0}\right)\right)\right)_{k=1}^{K_{i}} & \text { if } i \leq 29, \\
\left(\frac{1}{2}\left(L\left(t_{i, 0}\right)-L\left(t_{i, k}\right)\right)\right)_{k=1}^{K_{i}} & \text { if } i>29,
\end{array}\right)_{i=1}^{58} \in \mathbb{R}^{K_{1}} \times \mathbb{R}^{K_{2}} \times \cdots \times \mathbb{R}^{K_{58}} .
$$

We consider on $(0, \infty) \times(0, \infty)$ the uniform probability density $\pi_{0}$ for $(0,250) \times(0.0000001,25)$ as priori density. Updating this priori density by following Bayes' Theorem of Inverse Problems, see, e.g., Section 8.1 from [14] and Sections 2.8 and 6.2 from [15], we get the posterior probability density $\pi_{n e w}$ on $(0, \infty) \times(0, \infty)$ defined by

$$
\pi_{\text {new }}\left(c_{1}, c_{2}\right)=\frac{\ell\left(c_{1}, c_{2} \mid v^{\mathrm{obs}}\right) \pi_{0}\left(c_{1}, c_{2}\right)}{\int_{0}^{\infty} \int_{0}^{\infty} \ell\left(c_{1}^{\prime}, c_{2}^{\prime} \mid v^{\mathrm{obs}}\right) \pi_{0}\left(c_{1}^{\prime}, c_{2}^{\prime}\right) \mathrm{d} c_{2}^{\prime} \mathrm{d} c_{1}^{\prime}}
$$

with $\ell\left(\left(c_{1}^{\prime}, c_{2}^{\prime}\right) \mid v^{\text {obs }}\right)$ being the likelihood that the parameter pair has the value $\left(c_{1}^{\prime}, c_{2}^{\prime}\right)$ if the output $v^{\text {obs }}$ is observed. To derive the forward function $\Psi_{c_{1}, c_{2}}:(0, \infty) \times(0, \infty)$ allowing to formulate the likelihood function, we use a method similar to the derivation of (5.1). We consider (4.5) with $u(t):=\tanh \left(c_{3} I(t)\right), t_{b}=t_{i, 0}$, $t_{b}=t_{i, 0}$, and $t_{c}=t_{i, k}$ for all $k=0, \ldots, K_{i}$. Using that $L\left(t_{i, k}\right)$ and $L\left(t_{i, 0}\right)$ are approximations of $\mathcal{P} \mathcal{I}_{c_{1}, c_{2}}[u]\left(t_{i, k}\right)$ and $\mathcal{P I}_{c_{1}, c_{2}}[u]\left(t_{i, 0}\right)$, we end up with the assumption that there is a sample $\gamma=\left(\left(\gamma_{i, k}\right)_{k=0}^{K_{i}}\right)_{i=1}^{58}$ of a random variable $\Gamma=\left(\left(\Gamma_{i, k}\right)_{k=0}^{K_{i}}\right)_{i=1}^{58}$ on $\mathbb{R}^{K_{1}+1} \times \mathbb{R}^{K_{2}+1} \times \cdots \times \mathbb{R}^{K_{58}+1}$ such that

$$
\begin{aligned}
& \psi_{c_{1}, c_{2}}\left(\frac{\left|\tanh \left(c_{3} I\left(t_{i, k}\right)\right)-\tanh \left(c_{3} I\left(t_{i, 0}\right)\right)\right|}{2}\right) \\
= & \frac{1}{2}\left\{\begin{array}{ll}
L\left(t_{i, k}\right)+\gamma_{i, k}-\left(L\left(t_{i, 0}\right)+\gamma_{i, 0}\right), & \text { if } i \leq 29, \\
L\left(t_{i, 0}\right)+\gamma_{i, 0}-\left(L\left(t_{i, k}\right)+\gamma_{i, k}\right), & \text { if } i>29,
\end{array} \quad \forall k \in\left\{0,1, \ldots, K_{i}\right\}, i \in\{1, \ldots, 58\} .\right.
\end{aligned}
$$

Now, defining the forward function $\Psi:(0, \infty) \times(0, \infty) \rightarrow \mathbb{R}^{K_{1}} \times \mathbb{R}^{K_{2}} \times \cdots \times \mathbb{R}^{K_{58}}$ by

$$
\Psi\left(c_{1}, c_{2}\right):=\left(\left(\psi_{c_{1}, c_{2}}\left(\frac{\left|\tanh \left(c_{3} I\left(t_{i, k}\right)\right)-\tanh \left(c_{3} I\left(t_{i, 0}\right)\right)\right|}{2}\right)\right)_{k=1}^{K_{i}}\right)_{i=1}^{58} .
$$


and

$$
\eta:=\left(\begin{array}{llc}
\left(\frac{1}{2}\left(\gamma_{i, 0}-\gamma_{i, k}\right)\right)_{k=1}^{K_{i}} & \text { if } \quad i \leq 29, \\
\left(\frac{1}{2}\left(\gamma_{i, k}-\gamma_{i, 0}\right)\right)_{k=1}^{K_{i}} & \text { if } \quad i>29,
\end{array}\right)_{i=1}^{58} \in \mathbb{R}^{K_{1}} \times \mathbb{R}^{K_{2}} \times \cdots \times \mathbb{R}^{K_{58}}
$$

we have

$$
\Psi\left(c_{1}, c_{2}\right)+\eta=v^{\mathrm{obs}}
$$

and that $\eta$ is a sample for $\widetilde{H}:=\left(\left\{\begin{array}{ll}\left(\frac{1}{2}\left(\Gamma_{i, 0}-\Gamma_{i, k}\right)\right)_{k=1}^{K_{i}} & \text { if } i \leq 29, \\ \left(\frac{1}{2}\left(\Gamma_{i, k}-\Gamma_{i, 0}\right)\right)_{k=1}^{K_{i}} & \text { if } i>29,\end{array}\right)_{i=1}^{58}\right.$ and that $v^{\text {obs }}$ is a sample for $\Psi\left(c_{1}, c_{2}\right)+\widetilde{H}$. Hence, we have to consider the likelihood function $\ell$ associated to function mapping $\left(c_{1}^{\prime}, c_{2}^{\prime}\right) \in$ $(0, \infty) \times(0, \infty)$ to the random variable $\Psi\left(c_{1}^{\prime}, c_{2}^{\prime}\right)+\widetilde{H}$.

In the following, we assume that the components

$$
\Gamma_{1,0}, \Gamma_{1,1}, \ldots, \Gamma_{1, K_{1}}, \Gamma_{2,0}, \Gamma_{2,1}, \ldots, \Gamma_{2, K_{2}}, \ldots, \Gamma_{58,0}, \Gamma_{58,1}, \ldots, \Gamma_{58, K_{58}}, \text { of } \Gamma
$$

are independent random variables. Moreover, we assume that we have some $\sigma>0$ such that $\Gamma_{1,1}, \Gamma_{1,1}, \ldots, \Gamma_{1, K_{1}}$, $\Gamma_{2,1}, \Gamma_{2,1}, \ldots, \Gamma_{2, K_{2}}, \ldots, \Gamma_{58,1}, \Gamma_{58,1}, \ldots, \Gamma_{50, K_{58}}$ have the distribution $N\left(0, \sigma^{2}\right)$ representing the measurement error, i.e., the error between $\mathcal{P I}_{c_{1}, c_{2}}[u]\left(t_{i, k}\right)$ and $L\left(t_{i, k}\right)$.

For $i \in\{1, \ldots, 58\}$, it is assumed that $\gamma_{i, 0}$ contains in addition to the measurement error between $\mathcal{P} \mathcal{I}_{c_{1}, c_{2}}[u]\left(t_{i, 0}\right)$ and $L\left(t_{i, 0}\right)$ also the error generated by evaluating (4.5) for $t_{b}=t_{i, 0}$ and not for $t_{b}$ being the time $t_{i}^{*}$ near to $t_{i, 0}$ such that the continuous function $I$ has the local extremum in $t_{i}^{*}$. Since $t_{i}^{*}$ may be different from $t_{i, 0},(4.5)$ only holds with some additional error term. Hence, we assume that $\Gamma_{1,0}, \Gamma_{2,0}, \ldots, \Gamma_{58,0}$ have the distribution $N\left(0,(2 \sigma)^{2}\right)$.

Now, one can show that $\widetilde{H}$ has the density $\rho^{*}: \mathbb{R}^{K_{1}} \times \mathbb{R}^{K_{2}} \times \cdots \times \mathbb{R}^{K_{58}} \rightarrow[0, \infty)$ satisfying

$$
\begin{aligned}
\rho^{*}\left(\left(\left(w_{i, k}\right)_{k=1}^{K_{i}}\right)_{i=1}^{58}\right)= & \left(\frac{2}{\sqrt{2 \pi \sigma^{2}}}\right)^{\sum_{i=1}^{58} K_{i}}\left(\prod_{i=1}^{58} \sqrt{\frac{1}{4 K_{i}+1}}\right) \\
& \exp \left(-\frac{2}{\sigma^{2}} \sum_{i=1}^{58}\left(\sum_{k=1}^{K_{j}} w_{i, k}^{2}-\left(\frac{1}{4}+K_{i}\right)^{-1}\left(\sum_{k=1}^{K_{j}} w_{i, k}\right)^{2}\right)\right) .
\end{aligned}
$$

For $\left(c_{1}^{\prime}, c_{2}^{\prime}\right) \in(0, \infty) \times(0, \infty)$ it holds that $\Psi\left(c_{1}^{\prime}, c_{2}^{\prime}\right)+\widetilde{H}$ has the density

$$
\mathbb{R}^{K_{1}} \times \mathbb{R}^{K_{2}} \times \cdots \times \mathbb{R}^{K_{58}} \ni\left(\left(w_{i, k}\right)_{k=1}^{K_{i}}\right)_{i=1}^{58} \mapsto \rho^{*}\left(\left(\left(w_{i, k}\right)_{k=1}^{K_{i}}\right)_{i=1}^{58}-\Psi\left(c_{1}^{\prime}, c_{2}^{\prime}\right)\right)
$$

such that we can define $\ell\left(\left(c_{1}^{\prime}, c_{2}^{\prime}\right) \mid\left(\left(w_{i, k}\right)_{k=1}^{K_{i}}\right)_{i=1}^{58}\right)$ to be the formulae on the right-hand side. 

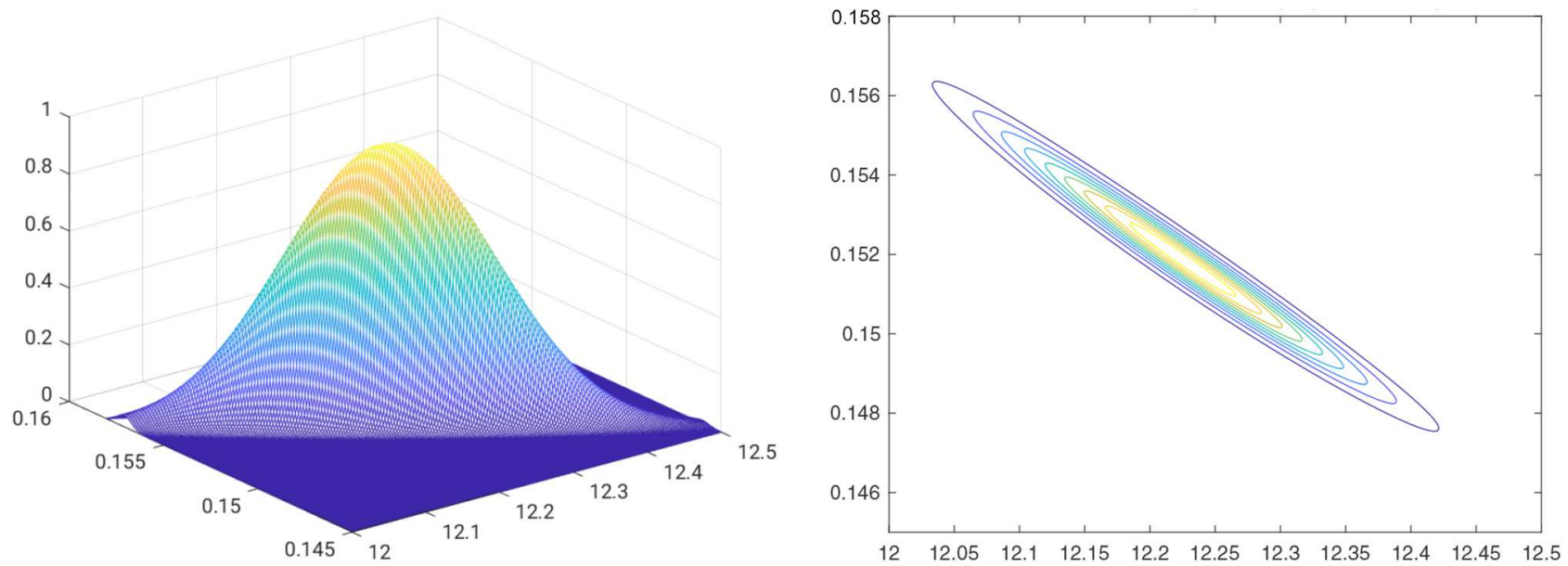

FIgURE 16. Normalized likelihood for the standard deviation 0.01 and 0.02 for the noise in the measured length. Left-hand side: 3D plot, right-hand side contour plot with isolines.

The normalized likelihood function $\ell_{\text {norm }}$ is defined by

$$
\ell_{\text {norm }}\left(\left(c_{1}^{\prime}, c_{2}^{\prime}\right) \mid\left(\left(w_{i, k}\right)_{k=1}^{K_{i}}\right)_{i=1}^{58}\right)=\frac{\ell\left(\left(c_{1}^{\prime}, c_{2}^{\prime}\right) \mid\left(\left(w_{i, k}\right)_{k=1}^{K_{i}}\right)_{i=1}^{58}\right)}{\max _{\left(c_{1}^{\prime \prime}, c_{2}^{\prime \prime}\right) \in(0, \infty)^{2}} \ell\left(\left(c_{1}^{\prime \prime}, c_{2}^{\prime \prime}\right) \mid\left(\left(w_{i, k}\right)_{k=1}^{K_{i}}\right)_{i=1}^{58}\right)} .
$$

We can replace the likelihood in (5.3) by the normalized likelihood without changing the result, also if we use a numerical approximation for the maximum instead of true maximum in the evaluation.

For $\sigma=0.01$ corresponding functions plots for $\ell_{\text {norm }}\left(\left(c_{1}^{\prime}, c_{2}^{\prime}\right) \mid v^{\text {obs }}\right)$ can be found in Figure 16, the position for numerical maximum for the likelihood is $\left(c_{1}, c_{2}\right)=(12.2253,0.1519)$. For $\sigma=0.05$ the corresponding functions plots are shown in Figure 17, the position for numerical maximum for the likelihood is the same as for $\sigma=0.01$.

Hence, we see that this likelihood function will generate a posterior probability density which is numerical equal to 0 except for a small region, such that the 58 computed pairs $\left(c_{1, i}, c_{2, i}\right)$ are obviously no typical samples for this probability density.

Recalling the derivation of the likelihood function and $[14,15]$, one realizes that this function is derived from a probability density for a noise disturbed output with using one parameter pair $\left(c_{1}, c_{2}\right)$ for all possible initial loading curves. Therefore, the Bayes' theorem generated a density on $(0, \infty) \times(0, \infty)$ that represents somehow the informations about the position of this one parameter pair that can be extracted by investigating the observed values in $v^{\text {obs }}$ and does not contain information for dealing with different parameter pairs for different subsets of the observation, i.e., this application of Bayes' theorem could not generated a density leading to the requested random variable $\left(C_{1}^{*}, C_{2}^{*}\right)$.

\subsection{Further research for UQ for magneto-mechanical component}

\subsubsection{UQ-issues for further research}

We would like to find a probability density on $(0, \infty) \times(0, \infty)$ such that the 58 computed pairs of parameter values or the appropriate subset of 26 parameter pairs introduced on Section 5.3 are typical samples for for this probability density. A further investigation of Bayes' theorem as discussed for example in [14, 15] yields that applying this theorem would require to use these probability densities as parameter instead of the number 

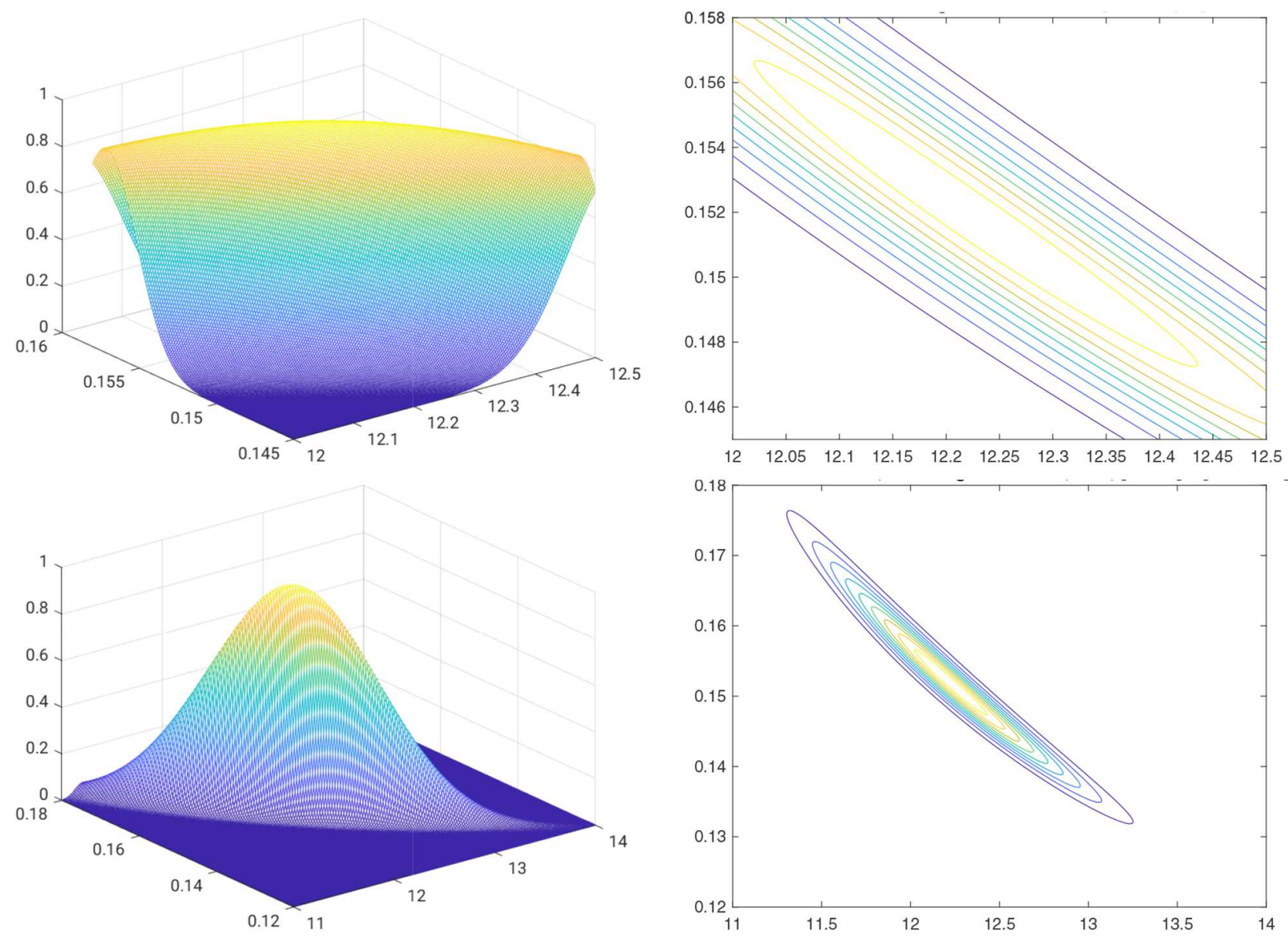

FIGURE 17. Normalized likelihood for the standard deviation 0.05 and 0.1 for the noise in the measured length. Left-hand side: 3D plot, right-hand side contour plot with isolines, top: input data range as in Figure 16, bottom: input data range adapted to considered likelihood function.

pairs $\left(c_{1}, c_{2}\right)$ considered in Section 5.4, and that Bayes' theorem would return a probability density over a set of probability density on $(0, \infty) \times(0, \infty)$ as result.

If one should apply now Bayes' theorem or try to determine the probability density on $(0, \infty) \times(0, \infty)$ maximizing the likelihood will be subject of further research.

\subsubsection{Modeling issues}

The considered data are derived from length change over magnetic field data. Hence, to be compatible with the thermodynamical consistent model derived in [4], one would need to replace the generalized Prandtl-Ishlinskiı operator $\mathcal{G}_{c_{1}, c_{2}, c_{3}}[H](t)$ by a function evaluation involving also the counter clockwise potential operator of an hysteresis operator appropriate to model the magnetization, e.g., the counter clockwise potential operator to $\mathcal{G}_{c_{1}, c_{2}, c_{3}}$. Corresponding investigations will be subject of further research.

\section{Conclusion}

The output of hysteresis operators depend on parameters, but their values may be not exactly known when modeling real world processes. 
These parameter can be considered as random variables and the methods of uncertainty quantification (UQ) allow to investigate the influence of the uncertainty on the output of the model and to determine the random variable representing the information on the parameter.

Acknowledgements. O. Klein would like to thank Prof. C. Schillings, University of Mannheim, for fruitful discussions.

\section{REFERENCES}

[1] M. Al Janaideh, C. Visone, D. Davino and P. Krejčí. The generalized Prandtl-Ishlinskii model: relation with the Preisach nonlinearity and inverse compensation error, in 2014 American Control Conference (ACC) June 4-6, 2014. Portland, Oregon, USA (2014).

[2] M. Al Janaideh, D. Davino, P. Krejčí and C. Visone, Comparison of Prandtl-Ishlinskii and Preisach modeling for smart devices applications. Phys. B: Condens. Matter 486 (2016) 155-159.

[3] M. Brokate and J. Sprekels, Hysteresis and phase transitions. Springer, New York (1996).

[4] D. Davino, P. Krejčí and C. Visone, Fully coupled modeling of magneto-mechanical hysteresis through 'thermodynamic' compatibility. Smart Mater. Struct. 22 (2013) 9.

[5] D. Davino and C. Visone, Rate-independent memory in magneto-elastic materials. Discrete Continuous Dyn. Syst. Ser. S 8 (2015) 649-691.

[6] O. Klein and P. Krejčí, Outwards pointing hysteresis operators and asymptotic behaviour of evolution equations. Nonlinear Anal. Real World Appl. 4 (2003) 755-785.

[7] O. Klein and P. Krejčí, Asymptotic behaviour of evolution equations involving outwards pointing hysteresis operators. Phys. B 343 (2004) 53-58.

[8] M. Krasnosel'skii and A. Pokrovskii, Systems with Hysteresis. Russian edition: Nauka, Moscow, 1983. Springer-Verlag, Heidelberg (1989).

[9] P. Krejčí, Hysteresis, Convexity and Dissipation in Hyperbolic Equations, Vol. 8 of Gakuto Int. Series Math. Sci. 63 Appl. Gakkōtosho, Tokyo (1996).

[10] W. Liu, A Geostatistical Approach toward Shear Wave Velocity Modeling and Uncertainty Quantification in Seismic Hazard. Dissertations, Clemson University (2018).

[11] S.F. Masri, R. Ghanem, F. Arrate and J. Caffrey, Stochastic nonparametric models of uncertain hysteretic oscillators. AIAA J. 44 (2006) 2319-2330.

[12] I.D. Mayergoyz, Mathematical Models of Hysteresis and their Applications. 2nd edn. Elsevier, Amsterdam (2003).

[13] D.D. Rizos and S.D. Fassois, A-posteriori identifiability of the maxwell slip model of hysteresis. IFAC Proc. 44 (2011) 10788-10793.

[14] R.C. Smith, Uncertainty quantification: theory, implementation, and applications, Vol. 12 of Computational Science \& Engineering. Society for Industrial and Applied Mathematics (SIAM), Philadelphia, PA (2014).

[15] T.J. Sullivan, Introduction to uncertainty quantification, Vol. 63 of Texts in Applied Mathematics. Springer, Cham (2015).

[16] S.P. Triantafyllou and E.N. Chatzi, A hysteretic multiscale formulation for validating computational models of heterogeneous structures. J. Strain Anal. Eng. Des. 51 (2015) 46-62.

[17] A. Visintin, Differential Models of Hysteresis, Vol. 111 of Applied Mathematical Sciences. Springer, New York (1994).

[18] C. Visone and M. Sjöström, Exact invertible hysteresis models based on play operators. Phys. B: Condens. Matter 343 (2004) $148-152$.

[19] Y. Zhang, Stochastic responses of multi-degree-of-freedom uncertain hysteretic systems (2011). 